\title{
Microstructure and Macromechanical Properties of Retaining Structure of Near-Water Reinforced Soil under Dry-Wet Cycle
}

\author{
Yujie Hou $\mathbb{D}^{1},{ }^{1}$ Bo Wang, ${ }^{1}$ Liang Huang $\left(\mathbb{D},{ }^{2}\right.$ Jianguo Xu, ${ }^{1}$ Dun Liu $\left(\mathbb{D},{ }^{1}\right.$ and Jiahua Zhu ${ }^{1}$ \\ ${ }^{1}$ School of Water Conservancy Engineering, Zhengzhou University, Zhengzhou 450001, China \\ ${ }^{2}$ School of Civil Engineering, Zhengzhou University, Zhengzhou 450001, China \\ Correspondence should be addressed to Liang Huang; ansys10@126.com and Dun Liu; liudun20202@163.com
}

Received 26 November 2020; Revised 13 January 2021; Accepted 7 February 2021; Published 19 February 2021

Academic Editor: Jianguo Wang

Copyright (c) 2021 Yujie Hou et al. This is an open access article distributed under the Creative Commons Attribution License, which permits unrestricted use, distribution, and reproduction in any medium, provided the original work is properly cited.

Reinforced soil-retaining structures that have been working in near-water environments for a long time are likely to affect their own mechanical properties due to the dry-wet cycle caused by changes in water level. In response to this problem, this paper uses a combination of macro- and microtests, selecting reinforced soil samples with four water content conditions, five overburden pressure conditions, three sets of dry-wet cycle conditions, and a total of 60 working conditions for testing. Scanning electron microscopy was used to observe the microscopic characterization of the reinforced soil particles under different times of the drywet cycle, and the pull-out test was used to study the mechanical properties of the interface of the reinforced materials and soils. The analysis results of the test show that the dry-wet cycles increase the porosity of the reinforced soil and the number of pores, among which the proportion of micro and small pores increases, the abundance and fractal dimension of reinforced soil particles increase, and the roughness of the particle surface is reduced. The change of the microstructure of the reinforced soil causes the cohesion of the soil to decrease in the macroscopic view. The friction coefficient and the ultimate pull-out force of the interface between the reinforced materials and the soils decrease with the increase of times of dry-wet cycle.

\section{Introduction}

Disaster investigations show that the dry-wet cycle can easily cause cracks on the surface of the near-water bank slope, and the soil will collapse and become unstable due to the decrease in strength. Landslides are the most typical type of geological disasters caused by the dry-wet cycle. After the completion of the Three Gorges Project, the reservoir water periodically rises and falls between the $145 \mathrm{~m}$ flood control limit water level and the $175 \mathrm{~m}$ normal water storage level every year, which revives ancient landslides in the reservoir area and increases new geological disasters. Figure 1 shows some examples of geological disasters in the Three Gorges reservoir area. The dry-wet cycles cause the deterioration of the mechanical properties of the near-water bank slope soil structure, and the resulting damage is often characterized by multiple, reciprocating, and long-lasting characteristics. Therefore, it is very necessary to study the degradation of soil structure mechanical properties caused by dry-wet cycles.
At present, the research on the performance of soil under the action of dry-wet cycles mainly includes soil-water characteristics, strength and deformation characteristics, crack development rules, and changes in the permeability of soil. In terms of soil-water characteristics [1-10], various studies have shown that the degradation of the mechanical properties of geotechnical structures by dry-wet cycles has the effect of reducing soil strength and decreasing soil cohesion.

Reinforced soil structure has good stability in actual engineering [11], and has received extensive attention in geotechnical engineering. Scholars have conducted comparative studies on the performance of reinforced soil and unreinforced soil. The results show that soil reinforced by geogrid exhibited higher bearing capacity than the unreinforced status, and can play roles in strengthening the levee [12-15]. During the long-term service of the near-water reinforced soil support structure, due to the difference in the application environment, it will encounter repeated soil- 


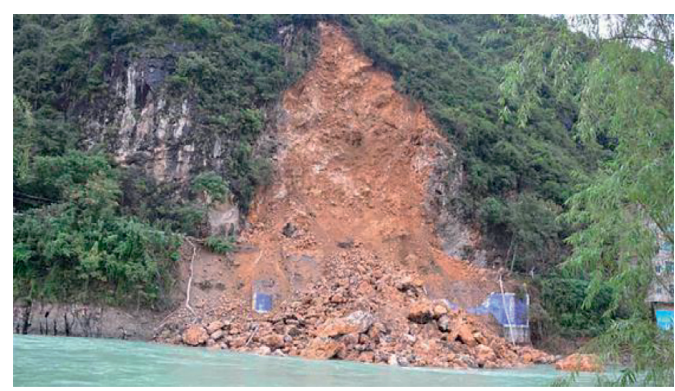

(a)

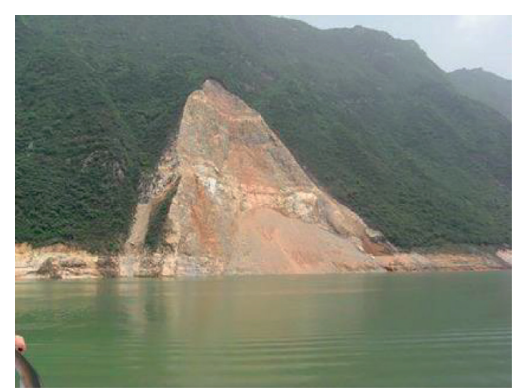

(b)

Figure 1: Landslide disaster in the Three Gorges reservoir area.

water interactions such as precipitation infiltration and river water level fluctuations. Although the performance is better than ordinary soil structure, the structural stability of the reinforced soil itself will still be affected under the action of water circulation, which will cause hidden danger to project safety. In 2013, a $26 \mathrm{~m}$ high, four-tier, geogrid-reinforced structure collapsed due to two sequential typhoon events with a total accumulated rainfall of more than $600 \mathrm{~mm}$. The failure mechanism and factors triggering the slope failure were examined [16]. Changes in groundwater level have effects on the stability of a geogrid-reinforced slope on loose marine soils in Qeshm Island, Iran. A series of geotechnical laboratory and in situ tests were used to study the factors affecting the stability of the embankment [17]. Therefore, studying the microstructure and the mechanical properties of reinforced soil under the action of dry-wet cycles is of great significance to engineering.

The dry-wet cycle process will cause the deterioration of the mechanical properties of the soil. The weakening of rock and soil parameters is a slow and gradual process. The damage effect may occur after long-term repeated action, and it would result in the collapse of the reservoir bank slope. However, the existing research on the performance degradation of near-water reinforced soil slopes lacks experimental data. And, this is why the dry-wet cycle test of reinforced soil is urgently needed. In this study, the scanning electron microscope observation and pull-out tests on reinforced soil under dry-wet cycle were conducted. And the influence of the times of dry-wet cycle on the microstructure changes of the reinforced soil and the mechanical properties of the reinforced soil interface were studied.

\section{Test Plan}

2.1. Test Equipment. In this experiment, microstructure research uses the Beijing Zhongke Instrument KYKYEM6200 scanning electron microscope to observe and image the microscopic morphology of the reinforced soil that has undergone different dry-wet cycles. The microstructure of the reinforced soil changes with times of the dry-wet cycle by analyzing the imaging pictures of the reinforced soil. The drawing test mainly adopts the microcomputer controlled electrohydraulic servo universal testing machine WAW-300. The test equipment is shown in Figure 2.
The equipment parameters are shown in Tables 1 and 2.

2.2. Test Materials. The filler for the test was taken from silty clay in Zhengzhou City, and its physical and mechanical parameters are shown in Table 3. Before the test, a mesh screen with a small aperture $(2 \mathrm{~mm})$ was used to remove impurities such as grass branches and gravel mixed in the clay.

The glass fiber two-way geogrid was selected for the test, and the main technical indicators are shown in Table 4.

2.3. Test Conditions. The test conditions are shown in Table 5. According to the setting of the test times of the relevant references on the study of the performance of the soilretaining structure $[18,19]$, the deterioration of soil properties changed from significant to insignificant after 7 times of the dry-wet cycle. The reinforcement of the geogrid allows the soil to maintain its performance under more times of the dry-wet cycle. At this stage, there are few tests on the reinforced soil structure with more than 20 times of the drywet cycle. Based on the above considerations, times of the dry-wet cycles designed for this test are 0,7 , and 30.

2.4. Test of the Dry-Wet Cycle. The test box is designed for the dry-wet cycle test of the reinforced soil. The side wall of the box has a narrow slit with a size of $210 \mathrm{~mm} \times 5 \mathrm{~mm}$ for the grid to lead out. The bottom of the test box is perforated. The pore diameter is $10 \mathrm{~mm}$. There are 20 in total, evenly distributed in the bottom of the test box. The model diagram of the test box is shown in Figure 3.

Before the dry-wet cycle test, the test-reinforced soil samples have to be processed. Use a mesh screen with a small aperture $(2 \mathrm{~mm})$ to remove impurities such as grass branches and gravel in the silty clay. Cut the glass fiber geogrid to a size of $30 \mathrm{~mm} \times 20 \mathrm{~mm}$.

Carry out the dry-wet cycle test on the reinforced soil. The dry-wet cycle adopts the process of soaking and drying: put the geotextile at the bottom of the test box, put the reinforced soil in the test box and compact it, and then immerse the test box in the water tank, with a soaking time of $2 \mathrm{~h}$. The test box is taken out of the water tank with 2 hours standing, and then the reinforced soil is dried. The drying process adopts a large dryer-the temperature of the dryer is 


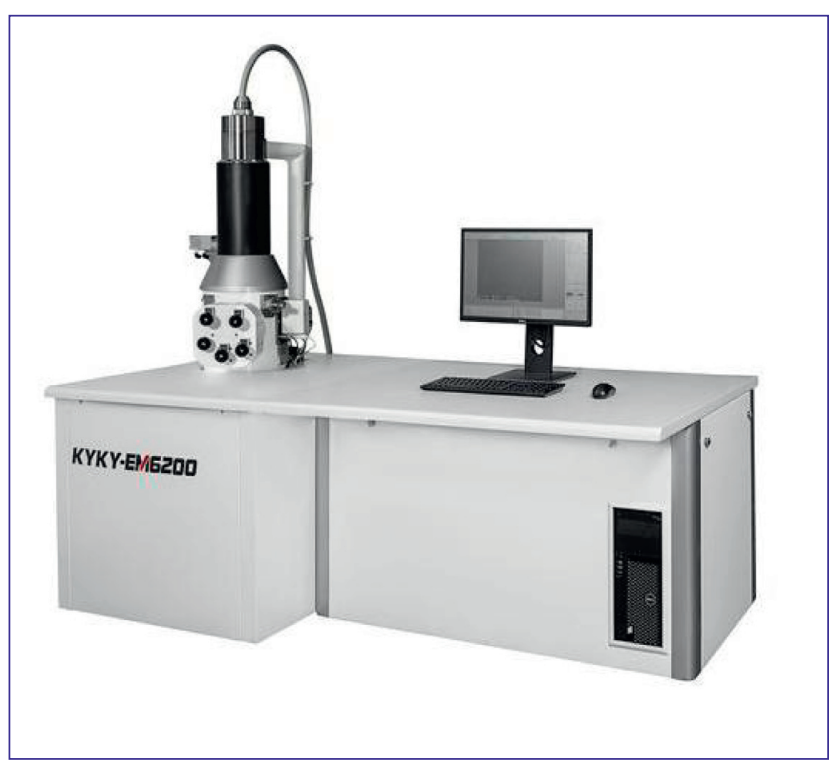

(a)

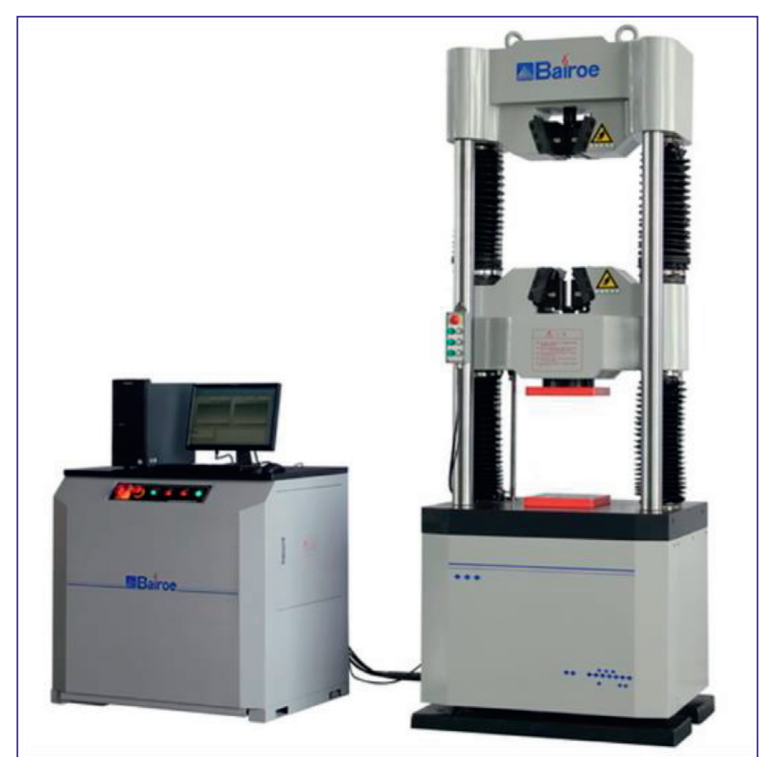

(b)

Figure 2: Test equipment. (a) Scanning electron microscope. (b) Universal testing machine.

TABle 1: Parameters of the KYKY-EM6200 scanning electron microscope.

\begin{tabular}{lc}
\hline Parameter & Value \\
\hline Resolution & $4.5 \mathrm{~mm}(30 \mathrm{kv})$ \\
Amplification & $15 \times \sim 25000 \times$ \\
Electron gun & Forked tungsten cathode \\
Accelerating voltage & $0 \sim 30 \mathrm{kv}$ \\
Lens system & Three-stage electromagnetic lens \\
Sample stage & Manual \\
Detector & Secondary electron detector \\
\hline
\end{tabular}

TABLe 2: Parameters of the universal testing machine WAW-300.

\begin{tabular}{lc}
\hline Parameter & Value \\
\hline Maximum test force & $300 \mathrm{kN}$ \\
Force resolution & $\pm 500000 \mathrm{bits}$ \\
Accuracy of test force indication & $\pm 1 \%$ \\
Displacement measurement range & $0 \sim 250 \mathrm{~mm}$ \\
Displacement resolution & $0.006 \mathrm{~mm}$ \\
Accuracy of displacement & $\pm 0.5 \%$ \\
Maximum distance between stretching jaws & $650 \mathrm{~mm}$ \\
\hline
\end{tabular}

set to $50^{\circ} \mathrm{C}$, and the drying time is $24 \mathrm{~h}$. After the drying is completed, let it stand for $2 \mathrm{~h}$.

According to the test results of the soil-water content at the borrow site, the dry-wet cycle amplitude is controlled at $4 \%-20 \%$, and the dry-wet cycles are performed 0 times, 7 times, and 30 times, respectively. The dry-wet cycle process is shown in Figure 4.

In order to study the changes in the microstructure of the reinforced soil before and after the dry-wet cycles, and analyze the relationship between the microstructure of the reinforced soil and the times of the cycle, a scanning electron microscope was used to observe the microstructure of the reinforced soil after different times of the dry-wet cycle.
Obtain the reinforced soil samples that have passed 0 times, 7 times, and 30 times of dry-wet cycle for drying. Blow off the loose soil debris on the surface of the soil sample, paste the conductive glue on the soil sample, spray gold to make the soil sample conductive, and then put the gold-sprayed soil sample into a scanning electron microscope for observation at different magnifications. Figure 5 is the scanning electron microscope and the soil sample diagram.

2.5. Pull-Out Test. Reinforced soil samples after 0 times, 7 times, and 30 times of the dry-wet cycle were used for pull-out tests. Add silty clay that has undergone a dry-wet cycle test to the test box, compact it at every $20 \mathrm{~mm}$ height, and ensure a certain degree of compaction each time. When the silty clay is added and compacted to a height of $120 \mathrm{~mm}$, put the cut-out geogrid specimen into the test box. After accurate positioning, continue to add the silty clay and compact it in layers. After compacting to the specified height, level the top of the packing. A $315 \mathrm{~mm} \times 295 \mathrm{~mm} \times 5 \mathrm{~mm}$ pressure-bearing steel plate is placed horizontally on the top of the filler of the test box to uniformly apply normal stress to the concrete block loaded on it, and heavy objects are placed according to the requirements of 5 different overlying pressures. After standing for 2 hours, a pull test was performed. Two steel plates and bolts are used to fix the geogrid-one end of the steel wire rope is connected to the fixture, and the guide pulley is bypassed, and the other end of the steel wire rope is connected to the fixture of the universal testing machine. Install the guide pulley on the fixture of the universal testing machine, adjust the position of the test box, tighten the wire rope, and make the narrow slot of the test box and the guide pulley in the same horizontal direction. Start the universal testing machine and set the relevant parameters. The horizontal drawing force is controlled by the displacement rate set by the computer at a rate of $1 \mathrm{~mm} / \mathrm{min}$. When the drawing displacement reaches the set value $30 \mathrm{~mm}$, the test is stopped 
TABle 3: Physical and mechanical parameters of the filler.

\begin{tabular}{lcccccc}
\hline Filler & $\begin{array}{c}\text { Bulk density } \gamma \\
\left(\mathrm{kN} \cdot \mathrm{m}^{-3}\right)\end{array}$ & $\begin{array}{c}\text { Elastic modulus } E \\
(\mathrm{MPa})\end{array}$ & $\begin{array}{c}\text { Poisson's ratio } \\
\mu\end{array}$ & $\begin{array}{c}\text { Cohesion } c \\
(\mathrm{kPa})\end{array}$ & $\begin{array}{c}\text { Internal friction angle } \varphi \\
\left({ }^{\circ}\right)\end{array}$ & $\begin{array}{c}\text { Dilatancy angle } \phi \\
\left({ }^{\circ}\right)\end{array}$ \\
\hline $\begin{array}{l}\text { Silty } \\
\text { clay }\end{array}$ & 15 & 20 & 0.35 & 15 & 20 & 0 \\
\hline
\end{tabular}

TABLe 4: Main technical indicators of the geogrid.

\begin{tabular}{lccccc}
\hline Reinforced materials & \multicolumn{2}{c}{ Breaking strength $\mathrm{kN} / \mathrm{m}$} & \multicolumn{2}{c}{ Elongation at break } & Grid size $(\mathrm{mm} \times \mathrm{mm})$ \\
\hline \multirow{2}{*}{ EGA100-100 } & Radial & Weft direction & Radial & Weft direction & $25.4 \times 25.4$ \\
& 103.5 & 102.8 & 3.0 & 3.0 & 2 \\
\hline
\end{tabular}

and the data are saved. Repeat the steps for the next working condition to measure the interface characteristics and related parameters of the geogrid and the fill under different dry-wet cycles, different water content, and different overlying pressure conditions. The pull-out test of the reinforced soil is shown in Figure 6.

\section{Results and Analysis of Electron Microscope Image}

The test soil samples were magnified 250 times, 500 times, 1250 times, 2500 times, and 5000 times to obtain scanning electron microscope images. The scanning electron microscope images of the reinforced soil samples that have not been subjected to dry-wet cycles are shown in Figure 7. It can be seen from the scanning results of the electron microscope that there are pores with different pore sizes and different shapes between the reinforced soils that have not been dried, and are wet, but the pore area is small and the particles are in close contact. Among the five scanning electron microscope images, the images magnified by 250 times have smaller particles and pores, and the microstructure is not obvious; the images magnified by 2500 times and 5000 times have fewer particles and pores, and the image information is not enough to analyze the typical microstructure of the reinforced soil. Since the contact information and the skeleton structure of the particles in the 1250-times magnified image are clearer, the scanning electron microscope image magnified by 1250 times is selected for the qualitative analysis of the microstructure of the reinforced soil; the image magnified by 500 times has the most comprehensive information on the particles and pores; therefore, the scanning electron microscope image magnified by 500 times is selected for image processing for quantitative analysis of the microstructure of the reinforced soil. The photos of a reinforced soil sample that has not undergone a dry-wet cycle at different magnifications are shown in Figure 7.

\subsection{Qualitative Analysis of Microstructure of the Reinforced} Soil. Figure 8 is a scanning electron microscope image of a reinforced soil sample that has undergone 0 times, 7 times, and 30 times of the dry-wet cycle, at a magnification of 1,250 times. As the times of the dry-wet cycle increases, the grain and the pore structure of the reinforced soil have changed. After 7 times of the dry-wet cycle, the pores between the soil particles increased significantly, and the number of pores increased; although 30 times of the dry-wet cycle were slightly larger than that of 7 times of the dry-wet cycle, the increase was not very large. The contact form between the grains of the reinforced soil without the dry-wet cycle is mostly surface-surface contact or surface-edge contact. After the dry-wet cycle, the contact forms of the edge-edge contact or the point-edge contact have increased, and the contact area between the particles have become smaller. When there is no dry-wet cycle, there are a lot of aggregates on the surface of the reinforced soil particles. With the increase of the times of the dry-wet cycle, the number of aggregates decreases, and the larger aggregates become smaller. These changes can be attributed to the effect of moisture on the reinforced soil during the dry-wet cycle. The dry-wet cycle of the soil can be understood as the repeated migration of water between the pores of the soil. The process of water migration in the pores washes away the soil particles, dissolves part of the soluble clay minerals, and separates a lot of small soil debris from the surface of the particles. The pore size and the number gradually increase, and the contact area between the soil particles gradually decreases.

\subsection{Quantitative Analysis of Microstructure of the Reinforced} Soil. Comprehensively analyze the information contained in the scanning electron microscope images of the reinforced soil samples at different magnifications and select the electron microscope images with a magnification of 500 times to quantitatively analyze the microstructure of the reinforced soil. Use the Image Pro Plus 6.0 [20] software to analyze the microstructure image of the reinforced soil. First, use the ruler function to convert the basic unit of the image from pixel form to length form, and correct the image length ruler with reference to the original ruler of the image. Since the gray scale difference between the grains and the pores in the original image of the reinforced soil sample is not obvious, the brightness, contrast, and gamma value of the image are adjusted by software, and the image is binarized by selecting an appropriate threshold to make a clear distinction between particles and pores, which is convenient for the next quantitative analysis of the image [21]. Figure 9 shows the binarized images of the electron microscope photos of the reinforced soil with different times of the dry-wet cycle. The white area of the image is 
TABLE 5: Test conditions.

0

4

1

\begin{tabular}{|c|c|c|}
\hline & 20 & $\begin{array}{l}4 \\
5 \\
1 \\
2 \\
3 \\
4 \\
5\end{array}$ \\
\hline 30 & 14 & $\begin{array}{l}1 \\
2 \\
3 \\
4 \\
5 \\
1 \\
2 \\
3 \\
4 \\
5 \\
1 \\
2 \\
3 \\
4 \\
5 \\
1 \\
2 \\
3 \\
4 \\
5\end{array}$ \\
\hline
\end{tabular}




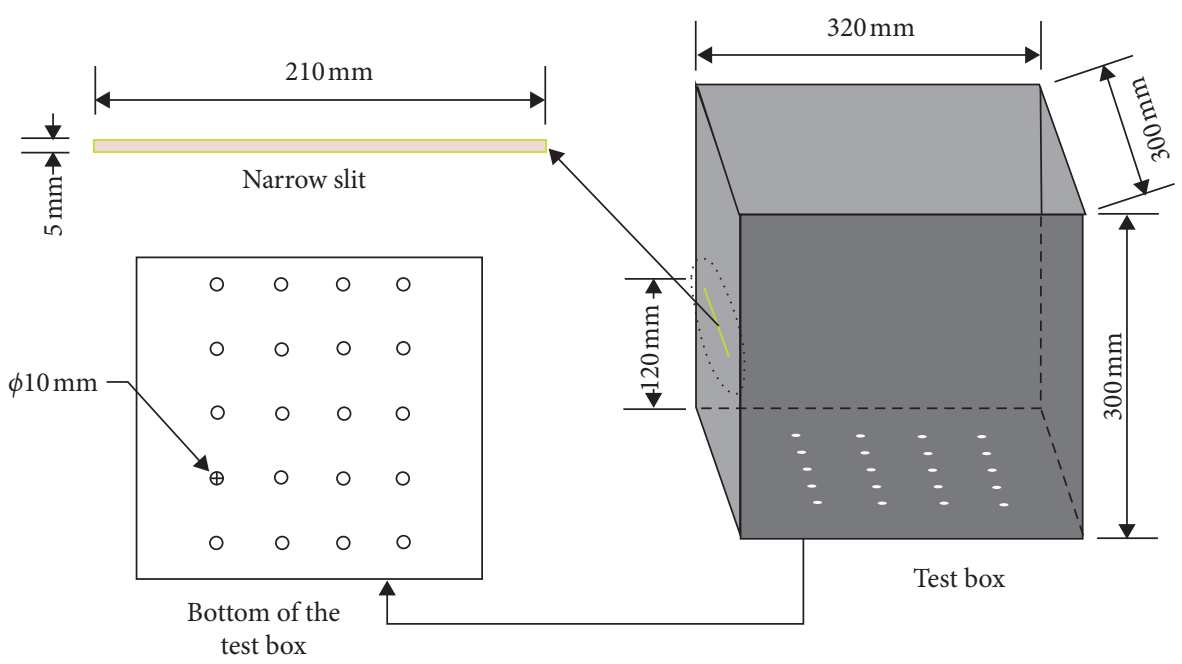

FIgURe 3: Model diagram of the test box.

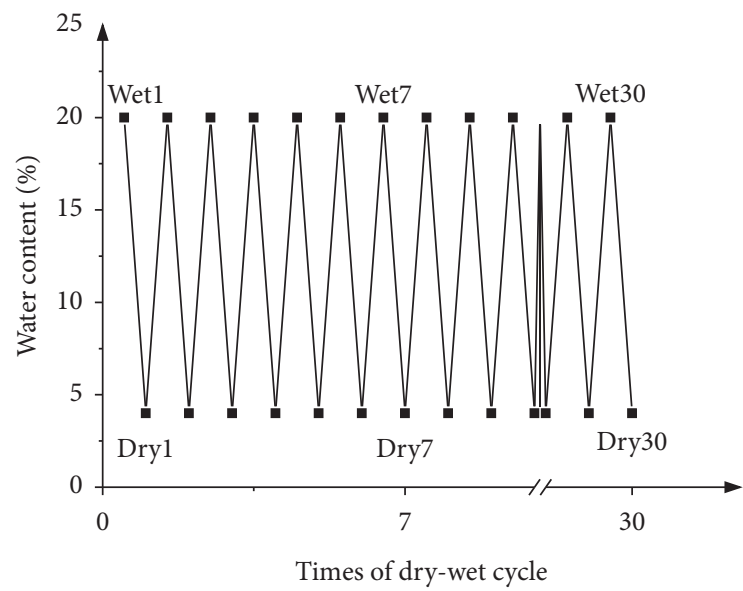

(a)

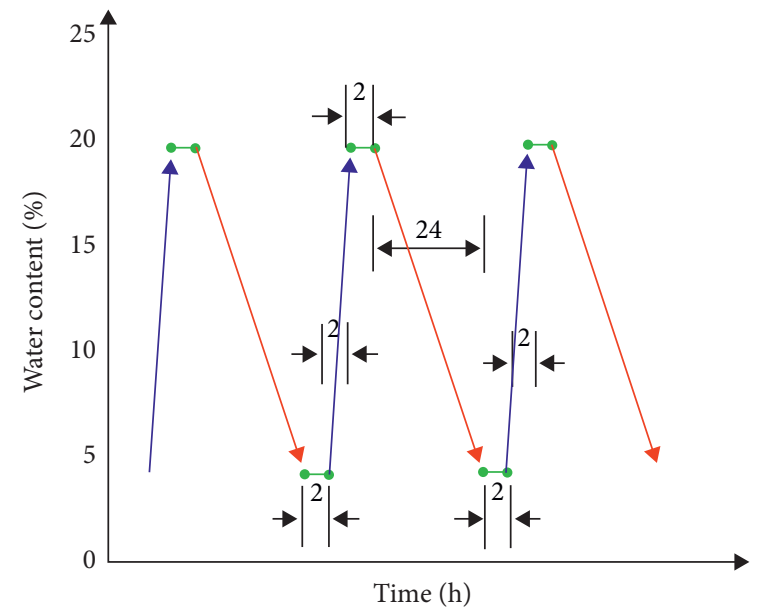

(b)

FIgURE 4: Schematic diagram of dry-wet cycle. (a) Times of dry-wet cycle. (b) One-Time of dry-wet cycle.

the particle part of the reinforced soil sample, and the black area is the pore part of the reinforced soil sample.

Quantitative analysis of the soil microstructure mainly studies the size and shape characteristic parameters of the soil particles or the unit bodies and the pores. According to research needs, this article mainly analyzes the porosity, pore size distribution, soil particle abundance, and the fractal dimension of reinforced soils with different times of the drywet cycle.

3.2.1. Porosity P. Use the Image Pro Plus 6.0 software to select the pore part of the microstructure image of the reinforced soil, and calculate the area of the pore part and the ratio of the area to the total area of the image. Particles and pores are more evenly distributed in the entire reinforced soil sample. The ratio of the area of the pores in the scanning electron microscope image to the total area of the image can be approximated as the porosity of the reinforced soil.

Figure 10 shows the variation of the porosity of the reinforced soil with the times of the dry-wet cycle. The porosity of the reinforced soil with different times of the drywet cycle measured by the software is: the porosity of the reinforced soil after 0 times of the dry-wet cycle is $9.22 \%$; the porosity of the reinforced soil after 7 times of the dry-wet cycle is $31.38 \%$; and the porosity of the reinforced soil after 30 times of the dry-wet cycle is $37.24 \%$.

With the increase of the times of the dry-wet cycle, the volume of the pores gradually increases under the action of water migration. After 7 times of the dry-wet cycle, the porosity increased significantly, and after the times of the dry-wet cycle increased to 30 , the amplitude increased slowly. It can be seen that after a certain times of the dry- 


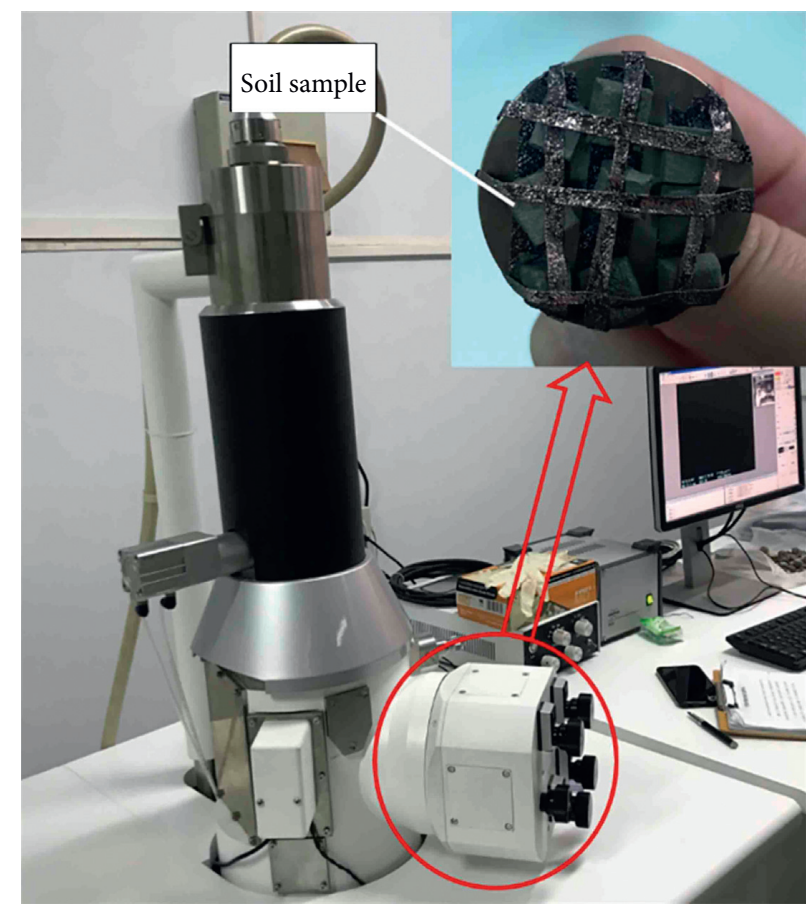

FIGURE 5: The scanning electron microscope and the soil sample diagram.

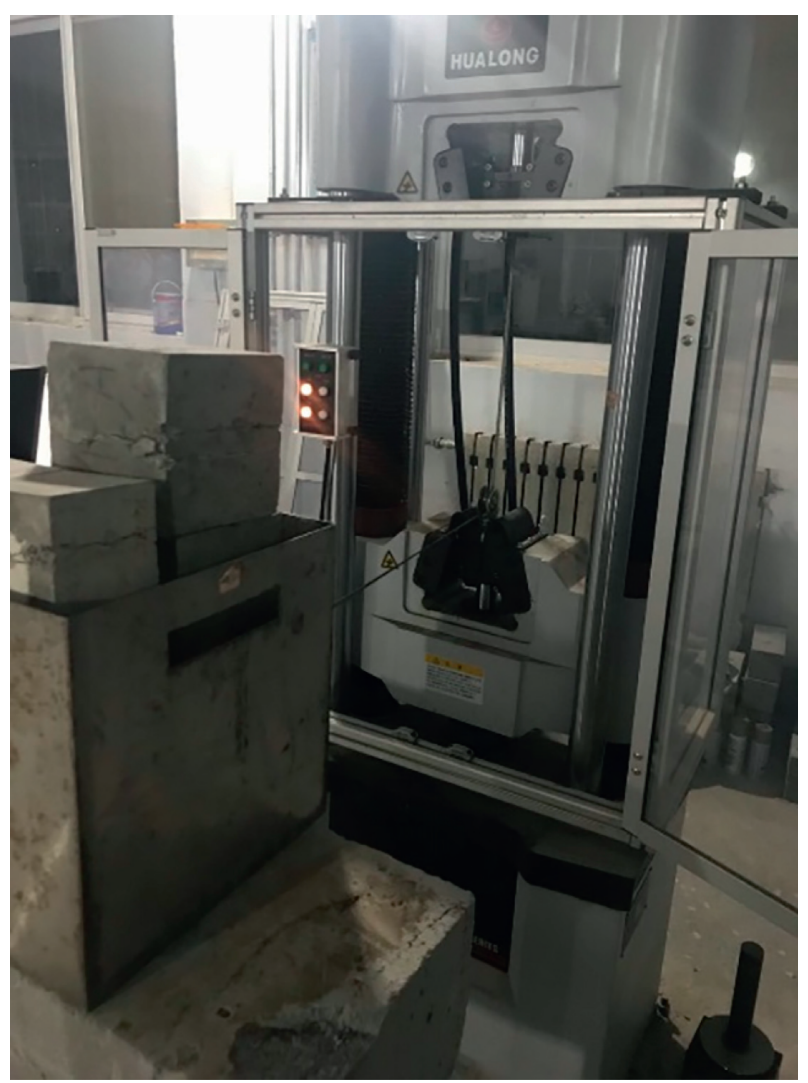

FIGURE 6: Pull-out tests of the reinforced soil.

wet cycle, the continuous increase of the times of the drywet cycle will not further increase the pore volume in the reinforced soil.
3.2.2. Average Pore Diameter $d$. The size of the pores in the soil is mostly measured by diameter, but the shape of the pore is actually quite complicated. Therefore, the diameter of a circle equal to the actual area of the pores is used to approximate the average diameter of the pores [22]. The calculation formula is

$$
d=\sqrt{\frac{4 S}{\pi}} .
$$

In the formula, $d$ is the average pore diameter, $\mathrm{mm}$; $S$ is the pore area, $\mathrm{mm}^{2}$.

It can be seen from Figure 10 that the pores with a pore diameter of less than $0.02 \mathrm{~mm}$ account for a larger proportion. Therefore, this paper combines the pore classification method proposed by Lei [23] based on the Mercury intrusion experiment to divide the pores of the reinforced soil into 4 categories according to the pore size:

Large pores, with a pore size greater than $0.016 \mathrm{~mm}$; medium pores, with a pore size between $0.004 \mathrm{~mm}$ and $0.016 \mathrm{~mm}$; small pores, with a pore size between $0.001 \mathrm{~mm}$ and $0.004 \mathrm{~mm}$; micro pores, with a pore size less than $0.001 \mathrm{~mm}$.

According to the software measurement results, draw a line-symbol graph of the relationship between the percentage of the pores with different pore diameters to the total pores under different times of the dry-wet cycle, which is used to analyze the changing law of pore diameter as the times of the dry-wet cycle increase. Figure 11 shows the variation of the four types of pores in reinforced soil under different times of the dry-wet cycle, and Figure 12 shows the variation of the percentage of the four types of pores in reinforced soil under different times of the dry-wet cycle. 


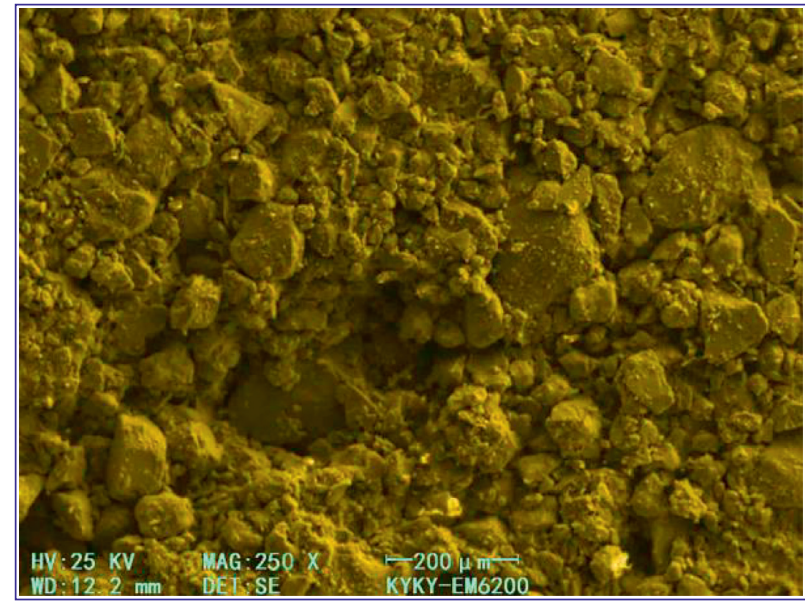

(a)

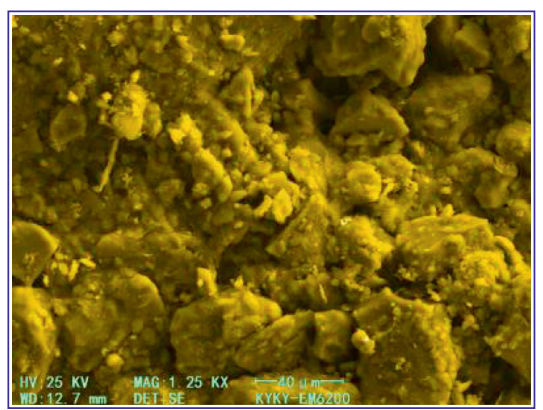

(c)

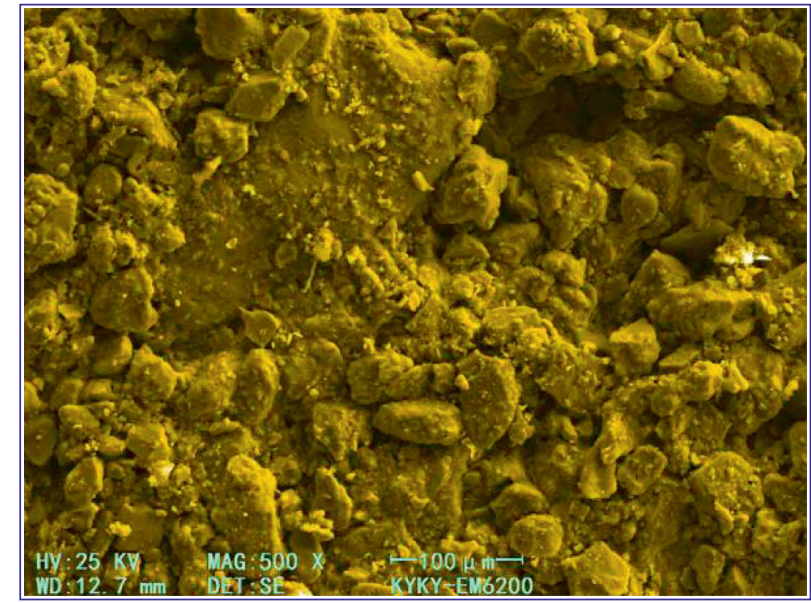

(b)

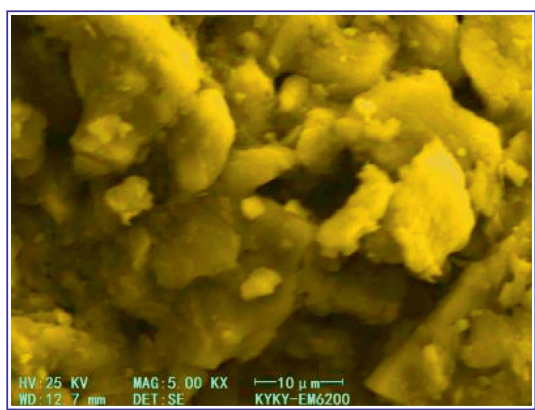

(e)

Figure 7: Microstructure of the reinforced soil with 0 times of the dry-wet cycle at different magnifications. (a) 250 times. (b) 500 times. (c) 1250 times. (d) 2500 times. (e) 5000 times.

It can be seen from the figure that as the dry-wet cycle progresses, the number of various pores in the sample shows an increasing trend. Among them, the absolute number of micropores increased the most, and the absolute number of medium pores increased the least. In the reinforced soil samples that have not undergone the dry-wet cycle, micropores accounted for $63 \%$ and small pores accounted for $28 \%$. The pores of the reinforced soil samples were mainly micropores and small pores. When the times of the dry-wet cycle increased to 7 , the number of the various pores increased. Among them, the number of the large and medium pores has the higher increase amplitude, thus the proportion of large and medium pores increased; the proportion of the small pores decreased, and the proportion of micropores increased. When the times of the dry-wet cycle increased to 30 , most of the new pores were micropores and small pores, and the proportion of micropores and small pores increased; the increase of large and medium pores slowed down, and the proportion decreased.

Analyze the variation of the percentage content of the various pores. When the soil has undergone a few times of the dry-wet cycle, water migrates between the pores of the soil and scours the particles, so that the micropores develop and expand, and then penetrate into large and medium pores; the scouring action produces new micropores. Therefore, the proportion of large and medium pores increases, the proportion of small pores decreases, and the proportion of micropores increases. After 30 times of the dry-wet cycle, the reinforced soil's weak water permeability and the effect of the geogrid limit the development of the pores, making the new pores mostly micropores and small pores. Under the action of 30 times of the dry-wet cycle, there are large and medium pores developed from micropores and small pores, and there are also micropores and small pores generated by refilling large and medium pores by some particles under the action of water. Therefore, although the absolute number of large and medium pores has increased, the increase amplitude is much lower than that of micropores and small pores, and their proportion in the number of pores has decreased.

3.2.3. Soil Particle Abundance C. The value obtained by dividing the short axis of the unit body of the particle by the long axis is the abundance [24], which can be expressed as the following:

$$
C=\frac{B}{L}
$$

$C$ is the abundance of particles, $B$ is the short axis of the unit body of the particle, $\mathrm{mm}$; $L$ is the long axis of the unit body of the particle, $\mathrm{mm}$. The abundance of soil particles is between 0 and 1 . The bigger the abundance $\mathrm{C}$ is, the soil particle is 


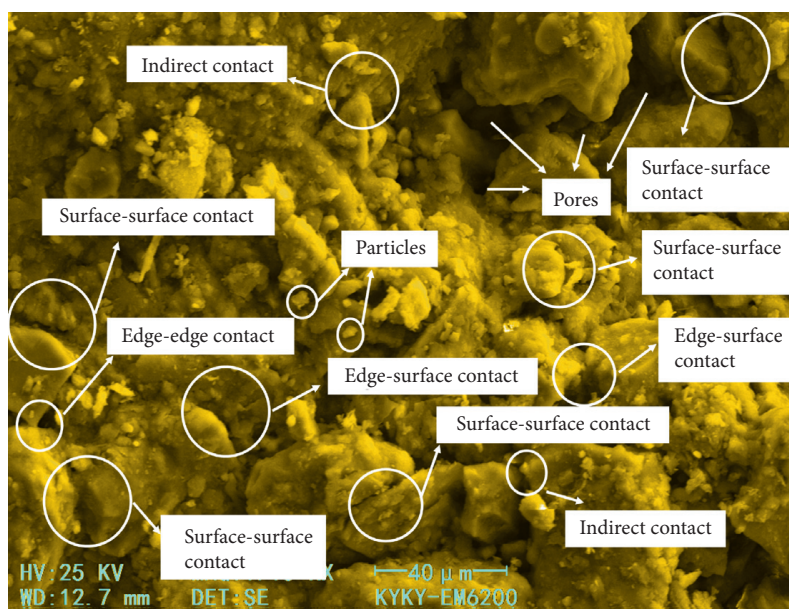

(a)

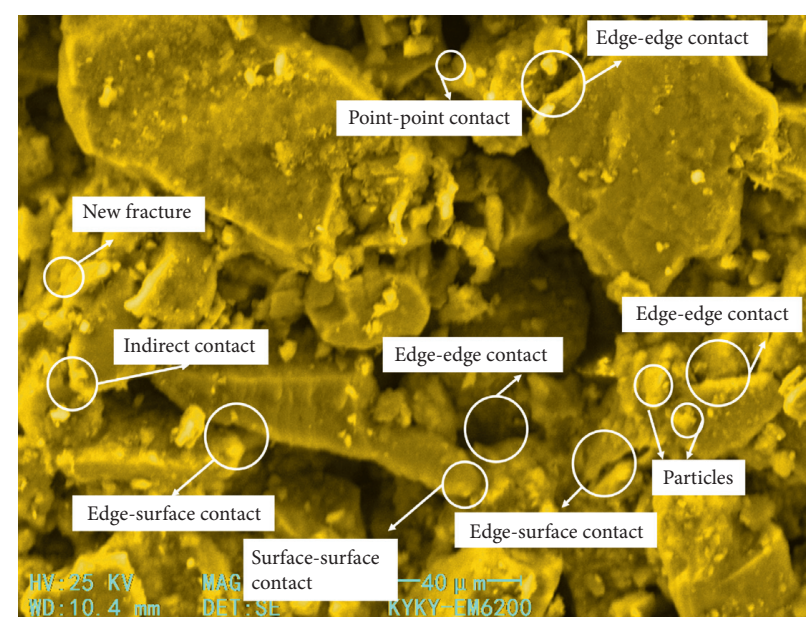

(b)

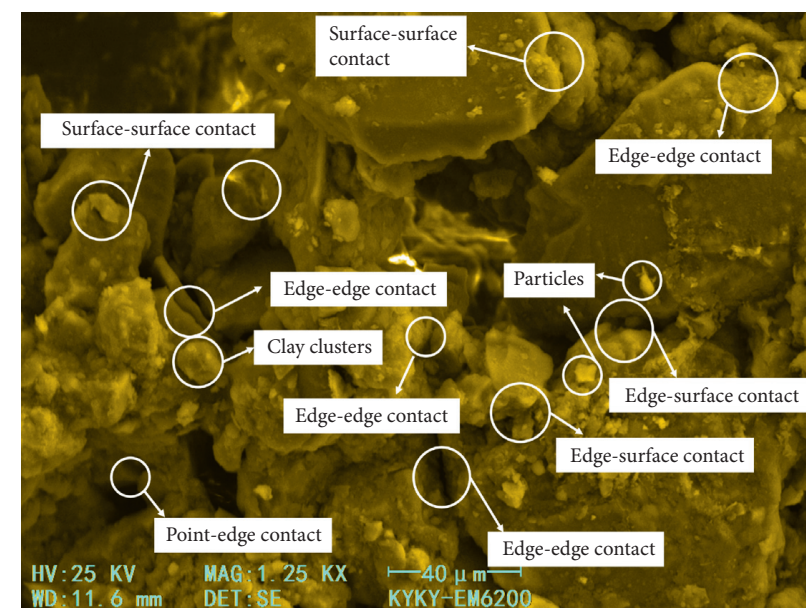

(c)

FiguRE 8: Image of reinforced soil with different times of the dry-wet cycle magnified by 1250 times. (a) 0 times of the dry-wet cycle. (b) 7 times of the dry-wet cycle. (c) 30 times of the dry-wet cycle.

much closer to a circle. Figure 13 shows the variation of the percentage content of the soil particles with different abundance under the action of the dry-wet cycle.

Analyze the percentage of the particles with various abundance under different times of the dry-wet cycle in the graph: without a dry-wet cycle, the soil particles with an abundance of 0.3 accounted for the most, 19.54\%; after 7 times of the dry-wet cycle, the soil particles with an abundance of 0.4 accounted for the most, $21.01 \%$; and after 30 times of the dry-wet cycle, the soil particles with an abundance of 0.5 accounted for the most, $20.27 \%$. The drywet cycle is repeated; the percentage of the soil particles with an abundance of $0.1-0.3$ gradually decreases, the percentage of the soil particles with an abundance of 0.4 first rises and then decreases, and the percentage of the soil particles with an abundance of 0.5-0.9 gradually rises. As the times of the dry-wet cycle increases, the abundance of the soil particles generally increases gradually. As the dry-wet cycle progresses, the soil particles are continuously washed away in the repeated migration of water. Some long particles with less abundance are cut into small particles with larger abundance, and more soil particles are rounded under the action of water erosion. The above reasons lead to the overall rise in soil particle abundance.

3.2.4. Fractal Dimension of the Soil Particles D. The equivalent area-perimeter method is used to calculate the fractal dimension of the shape distribution of the reinforced soil particles [25]. Moore and Donaldson [26] proposed a formula for calculating the fractal dimension of the shape distribution of the linear relationship between the equivalent perimeter and the equivalent area in logarithmic coordinates:

$$
\log (\text { Perimeter })=\frac{D}{2} \times \log (\text { Area })+C .
$$

Perimeter is the equivalent perimeter of the reinforced soil particle unit body, $\mathrm{mm}$; Area is the equivalent area of the particle unit body, $\mathrm{mm}^{2}$; $C$ is the fitting constant.

The fractal dimension $D$ mainly characterizes the roughness of the particle micro-interface. The larger the fractal dimension, the lower the roughness of the particle 


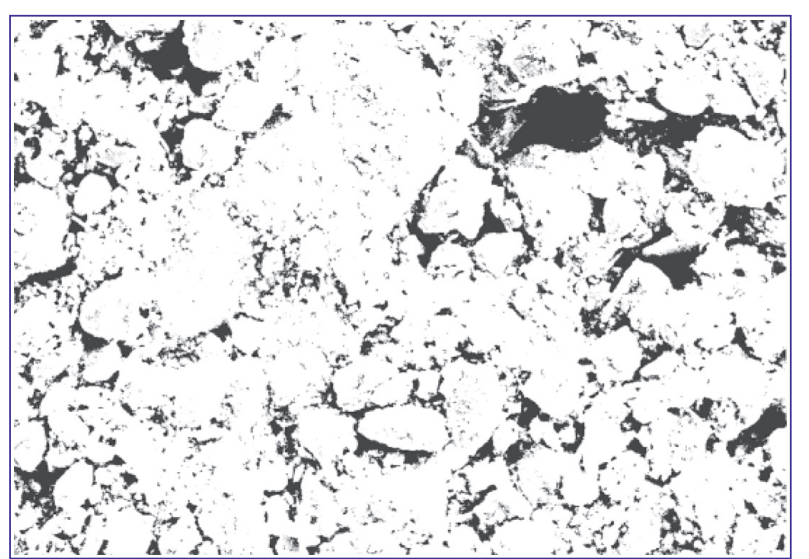

(a)

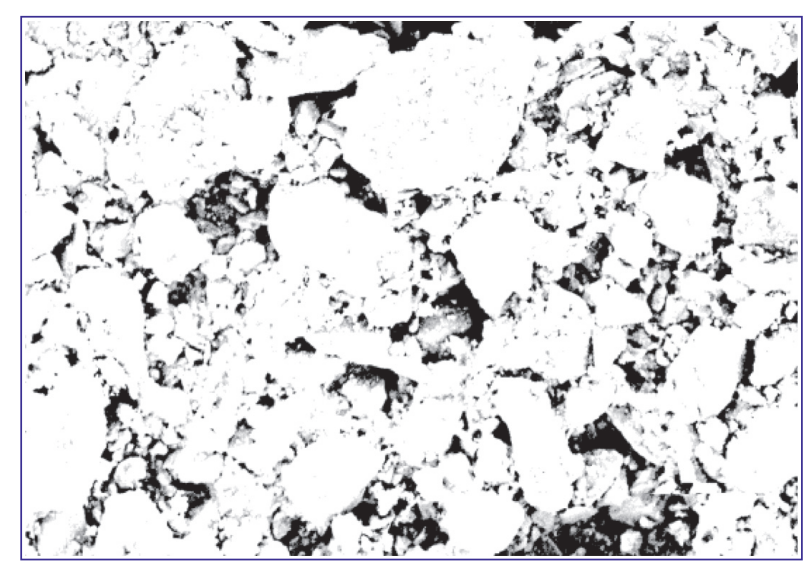

(b)

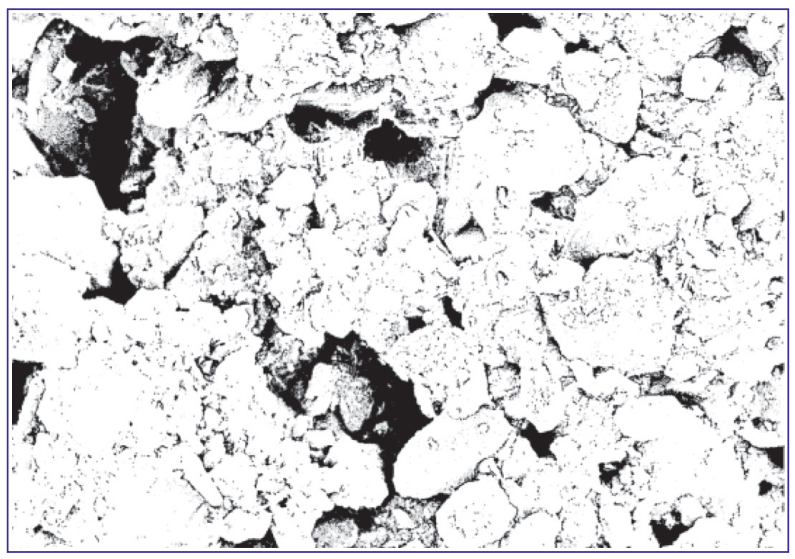

(c)

FIGURE 9: Binary processing of electron microscope images. (a) 0 times of the dry-wet cycle (b) 7 times of the dry-wet cycle. (c) 30 times of the dry-wet cycle.

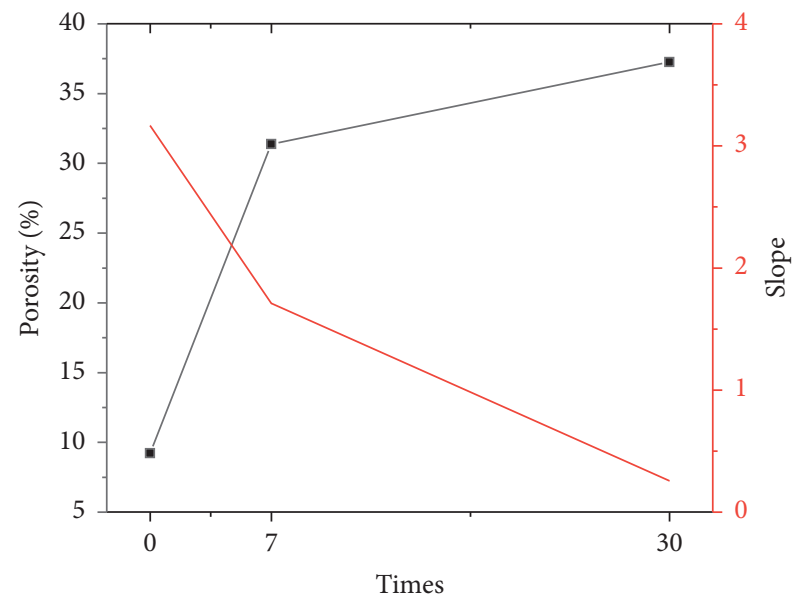

FIGURE 10: Change of porosity with the times of the dry-wet cycle.

interface; conversely, the higher the roughness. Figure 14 is the fractal dimension of the particles with different times of the dry-wet cycle. Times are calculated such that the fractal dimension of the reinforced soil particles without the drywet cycle is 1.1072, the fractal dimension of the reinforced soil particles after 7 times of the wet-dry cycle is 1.1466 , and

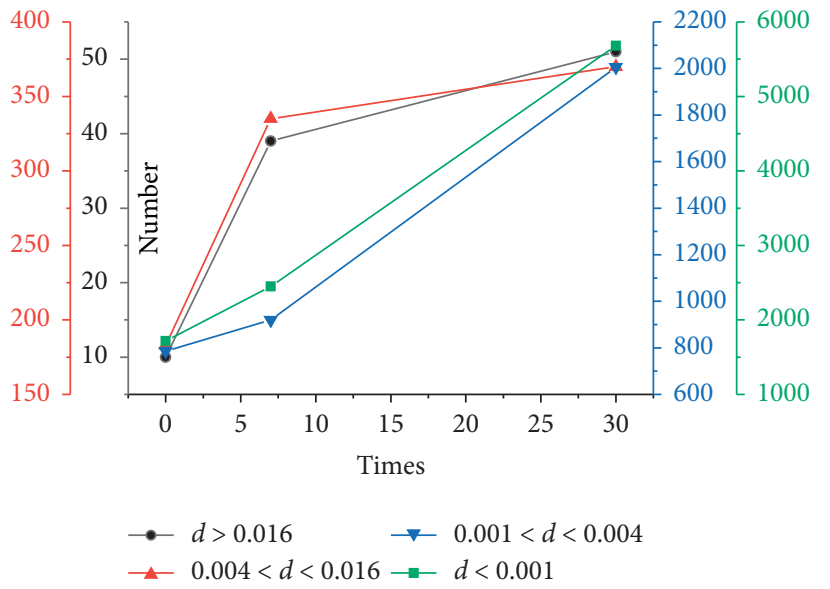

FIgURE 11: Variation of the number of pores under different times of the dry-wet cycle.

the fractal dimension of the reinforced soil particles after 30 times of the wet-dry cycle is 1.1954 .

With the increase in the times of the dry-wet cycle, the fractal dimension of the reinforced soil particles has an increasing trend but the change is not large. The effects of the 


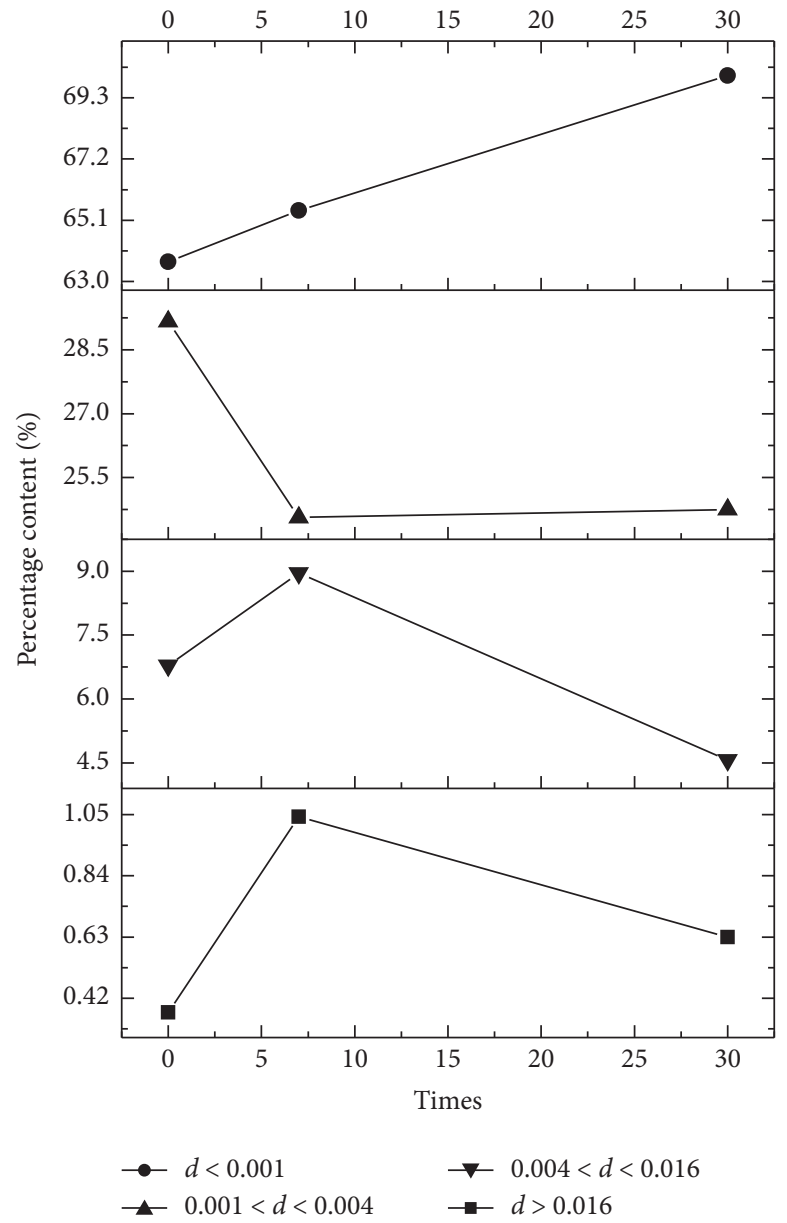

FiguRE 12: Variation of the percentage of pores under different times of the dry-wet cycle.

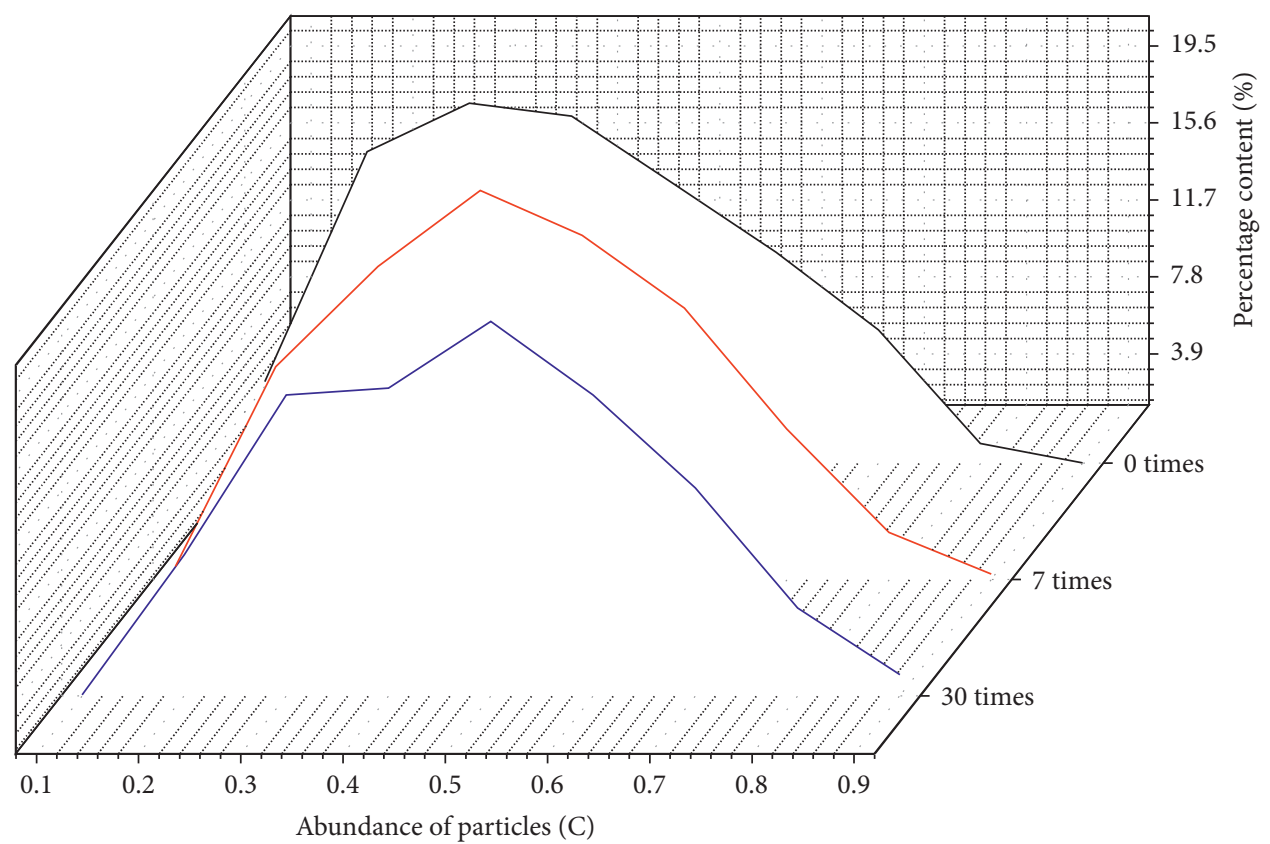

Figure 13: Variation of percentage content of the soil particles with different abundance under the action of the dry- wet cycle. 


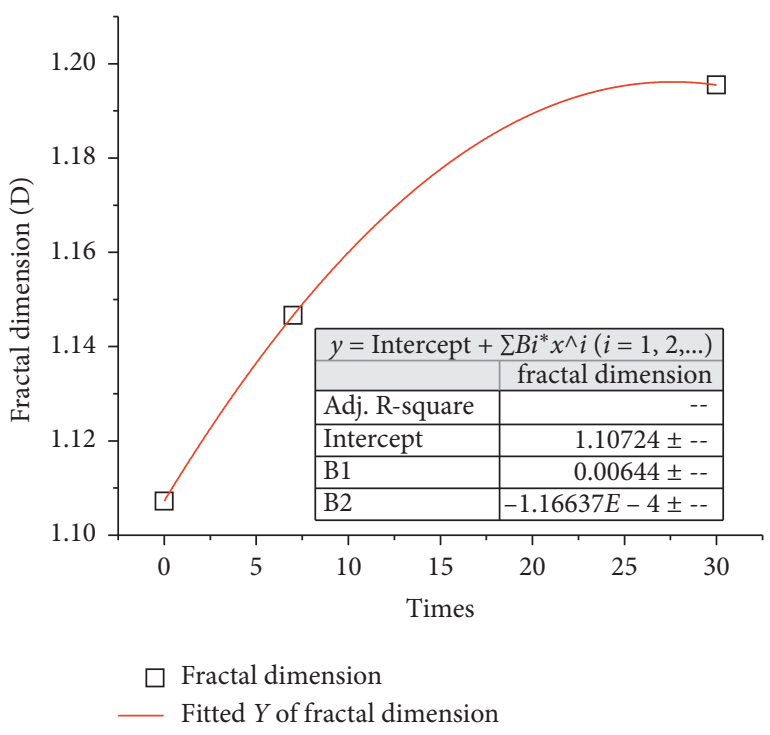

Figure 14: Fractal dimension of particles with different dry-wet cycle times.

multiple dry-wet cycles increase the number of small particles in the reinforced soil. The increase of small particles makes the overall shape and composition of the soil more complex; the migration of water reduces the surface roughness of the particles. These reasons lead to the increase of the fractal dimension of the reinforced soil particles.

\section{Analysis of Mechanical Properties of the Reinforced Soil}

\subsection{Analysis of Ultimate Pull-Out Force Variation.} Figure 15 is a graph showing the variation of the ultimate pull-out force of the reinforced soil with the overburden pressure under different times of the dry-wet cycle and different water content.

It can be seen from Figure 15 that when the water content and the times of the dry-wet cycle are constant, the ultimate pull-out force of the reinforced soil increases with the increase of the overburden pressure, and the overall trend is linearly increasing. This is due to the increase of the overburden pressure, and the frictional resistance of the interface between the geogrid and the fill also increases. When the water content and the overburden pressure are constant, the ultimate pull-out force of the reinforced soil decreases with the increase of the times of the dry-wet cycle. With a water content of $14 \%$ and an overburden pressure of $5 \mathrm{kPa}$, the ultimate pull-out force of the reinforced soil after 0 times of the dry-wet cycle is $0.9486 \mathrm{kN}$, and the ultimate pull-out force of the reinforced soil after 7 times of the dry-wet cycle is reduced by $3.86 \%$. After 30 times of the dry-wet cycle, the ultimate pull-out force of the reinforced soil is reduced by $5.3 \%$. After 7 times of the dry-wet cycle, the ultimate pull-out force of the reinforced soil decreased significantly, and after 30 times of the dry-wet cycle, the decrease of the ultimate pull-out force decreased. It shows that when times of the dry-wet cycle increase to more than 7, the times of the cycle has little effect on the ultimate pull-out force of the reinforced soil. Combined with the results of the scanning electron microscope images analysis, the dry-wet cycles' effect increases the porosity of the reinforced soil, reduces the contact area between the reinforced materials and soils, and reduces the bite force. These reasons cause the ultimate pull-out force of the reinforced soil to decrease as the times of the dry-wet cycle increases. After 7 times of the dry-wet cycle, the porosity of the reinforced soil increased significantly, and the ultimate pull-out force decreased. After 30 times of the dry-wet cycle, the porosity of the reinforced soil increased slightly, and the ultimate pull-out force decreased slightly too. The dry-wet cycle also increases the abundance of particles, the soil particles are rounded, and the bite force to the reinforcement decreases, which reduces the ultimate pull-out force.

4.2. Analysis of Shear Stress and Normal Stress. The maximum shear stress of the reinforced soil interface $\tau$ can be obtained by the ultimate pull-out force measured by the reinforced soil pull-out test, Assuming that the friction between the fill and the geogrid is evenly distributed, it can be solved by formula (4) [27]:

$$
\tau=\frac{T}{2 B L}
$$

$T$ is the ultimate pull-out force in the pull-out test of the reinforced soil, $\mathrm{kPa} ; B$ and $L$ are the width and the length of the contact surface between the geogrid and the fill, respectively, $\mathrm{m}$. In this pull-out test of the reinforced soil, $B=0.2 \mathrm{~m}, L=0.25 \mathrm{~m}$.

The normal stress $\sigma$ of the pull-out test can be solved by

$$
\sigma=P_{1}+P_{2}+\gamma h .
$$

$P_{1}$ is the overburden pressure exerted on the reinforced soil by the steel-bearing plate, $\mathrm{kPa} ; P_{2}$ is the dead weight of the steel-bearing plate, $0.2 \mathrm{kPa} ; \gamma$ is the bulk density of the reinforced soil. The corresponding bulk densities of the reinforced soils with water contents of $4 \%, 8 \%, 14 \%$, and $20 \%$ are $13.8 \mathrm{kN} / \mathrm{m}^{3}, 14.4 \mathrm{kN} / \mathrm{m}^{3}, 15.2 \mathrm{kN} / \mathrm{m}^{3}$, and $16 \mathrm{kN} /$ $\mathrm{m}^{3}$, respectively; $h$ is the height of the fill covered on the geogrid, $0.1 \mathrm{~m}$.

Through formulas (4) and (5), the shear stress and the normal stress can be obtained under the conditions of different times of the dry-wet cycle and different water content, and the relationship between shear stress and normal stress can be obtained by fitting. The relationship between shear stress and normal stress is expressed by

$$
\tau=\sigma \tan \varphi+c,
$$

$\varphi$ is the friction angle of the reinforced soil, ${ }^{\circ} ; c$ is the cohesion of the reinforced soil, $\mathrm{kPa}$. The relationship between shear stress and normal stress is shown in Table 6:

Figure 16 shows the relationship between the shear stress and the normal stress of the reinforced soil with different times of the dry-wet cycle.

It can be seen from Table 6 and Figure 16 that when the times of the dry-wet cycle are constant, the shear stress of the reinforced soils with different water contents is linearly related to the normal stress. When times of the drywet cycle is constant, the internal friction angle of the 

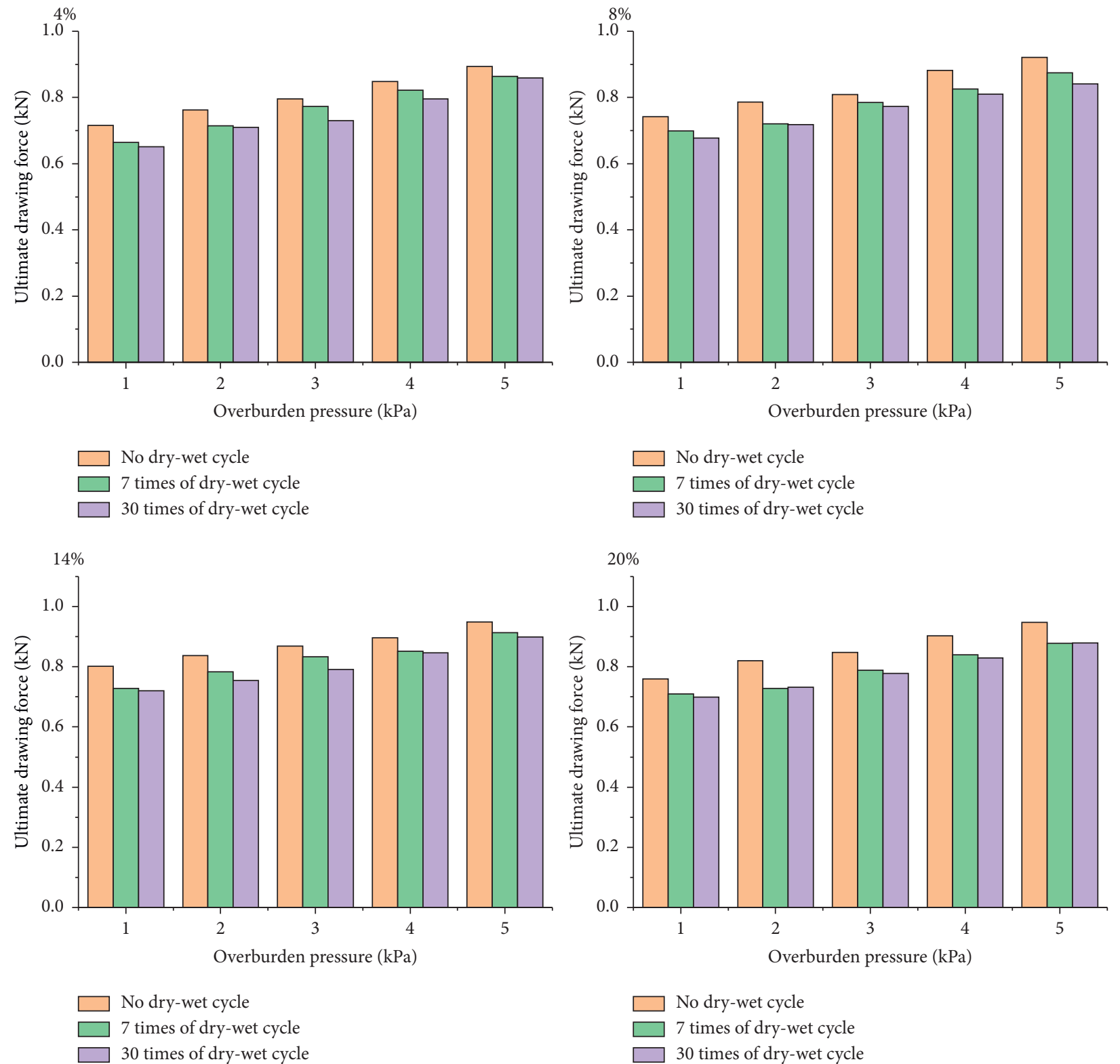

FIGURE 15: Variation of the ultimate pull-out force with overburden pressure for different times of the dry-wet cycle.

reinforced soil does not change significantly with the increase of water content; the cohesion of the reinforced soil first increases and then decreases, and the cohesion of the reinforced soil is the largest when the water content is $14 \%$. The linear difference of the relationship between the shear stress and the normal stress of the reinforced soil under different water content conditions does not change significantly with the increase of the times of the dry-wet cycle.

4.3. Analysis of Friction Coefficient Changes. The interface characteristics of the reinforced soil can be reflected by the apparent friction coefficient, which can reflect the interaction between the geogrid and the fill. The formula for calculating the apparent friction coefficient is [28]

$$
f=\frac{\tau}{\sigma}
$$

where $\tau$ and $\sigma$ are the maximum shear stress and the normal stress in the pull-out test of the reinforced soil, respectively, $\mathrm{kPa}$.

Figure 17 is a graph showing the change of the friction coefficient of the reinforced soil under different normal stresses and different cycle times.

It can be seen from Figure 17 that the times of the drywet cycle and the water content of the reinforced soil do not change the trend of the interface friction coefficient of the reinforced soil with the normal stress. When the times of the dry-wet cycle and the water content of the reinforced soil are constant, the interface friction coefficient decreases with the increase of the normal stress. When the times of the dry-wet 
TABLE 6: Relationship between shear stress and normal stress.

\begin{tabular}{|c|c|c|c|c|}
\hline Times of cycle (times) & Water content (\%) & Fitting formula & Internal friction angle $\left({ }^{\circ}\right)$ & Fit index \\
\hline \multirow{4}{*}{0} & 4 & $\tau=0.4435 \sigma+5.9983$ & 23.92 & 0.9947 \\
\hline & 8 & $\tau=0.4535 \sigma+6.1768$ & 24.39 & 0.9701 \\
\hline & 14 & $\tau=0.3536 \sigma+7.0370$ & 19.47 & 0.9843 \\
\hline & 20 & $\tau=0.4604 \sigma+6.3421$ & 24.72 & 0.9880 \\
\hline \multirow{4}{*}{7} & 4 & $\tau=0.5055 \sigma+5.3604$ & 26.82 & 0.9959 \\
\hline & 8 & $\tau=0.4562 \sigma+5.6888$ & 24.52 & 0.9789 \\
\hline & 14 & $\tau=0.4396 \sigma+6.1451$ & 23.73 & 0.9737 \\
\hline & 20 & $\tau=0.4480 \sigma+5.7380$ & 24.13 & 0.9753 \\
\hline \multirow{4}{*}{30} & 4 & $\tau=0.5015 \sigma+5.1941$ & 26.63 & 0.9693 \\
\hline & 8 & $\tau=0.4202 \sigma+5.6905$ & 22.79 & 0.9873 \\
\hline & 14 & $\tau=0.4475 \sigma+5.9104$ & 24.11 & 0.9844 \\
\hline & 20 & $\tau=0.4584 \sigma+5.6367$ & 24.63 & 0.9903 \\
\hline
\end{tabular}
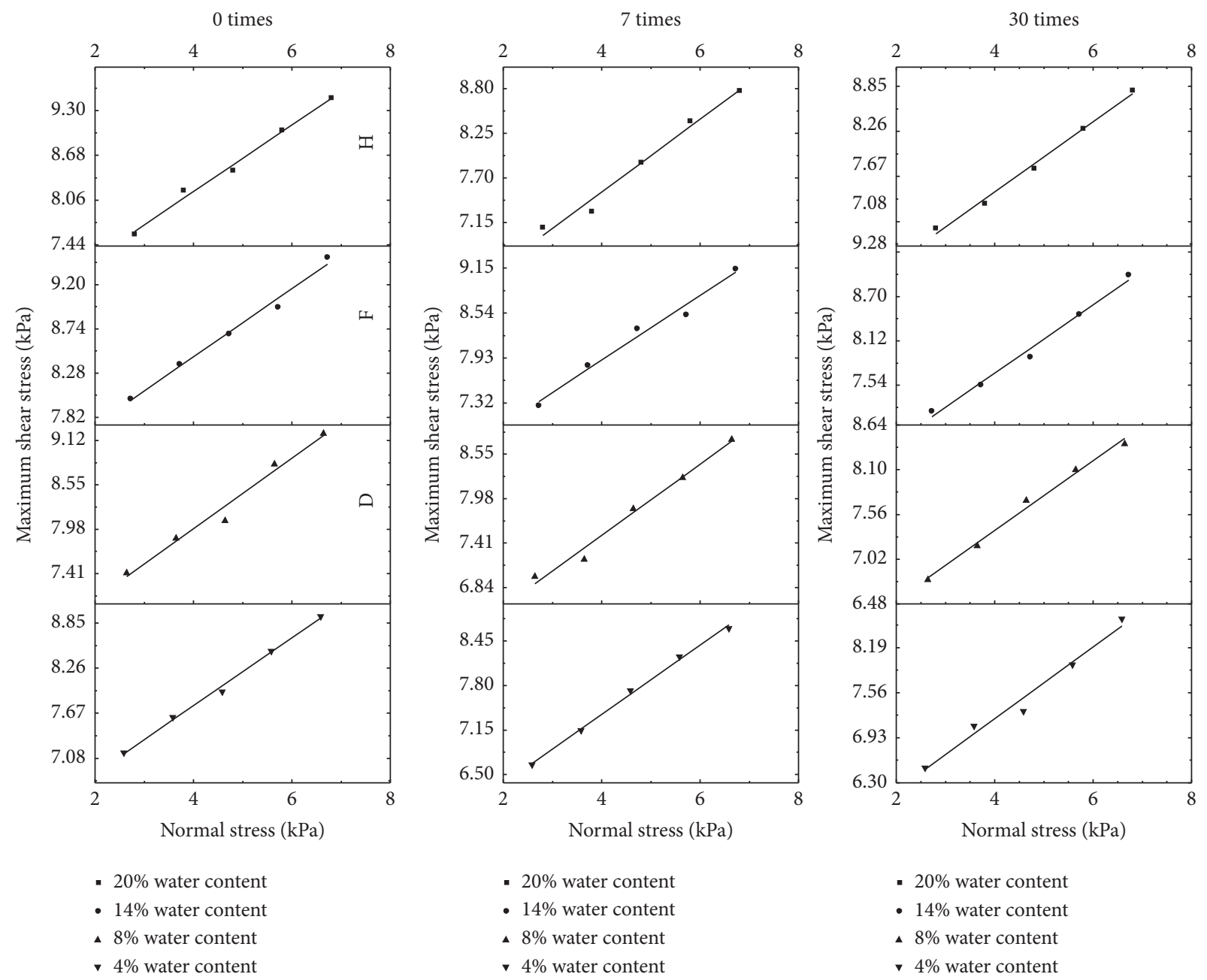

FIGURE 16: The relationship between the shear stress and the normal stress of the reinforced soil with different cycles.

cycle and the normal stress are constant, the corresponding water content of the reinforced soil interface friction coefficient from large to small is $14 \%, 20 \%, 8 \%$, and $4 \%$, respectively; when the times of the dry-wet cycle are constant, and the water content is $14 \%$, the interfacial friction coefficient of the reinforced soil is the largest when the normal stress is the smallest. The interfacial friction coefficients corresponding to 0,7 , and 30 times of the dry-wet cycle are $2.95,2.68$, and 2.65 , respectively.

Figure 18 is a graph showing the variation of friction coefficient at the interface of $14 \%$ water content at the different cycle times.

It can be seen from Figure 18 that when the normal stress and the water content are constant, the interface friction 


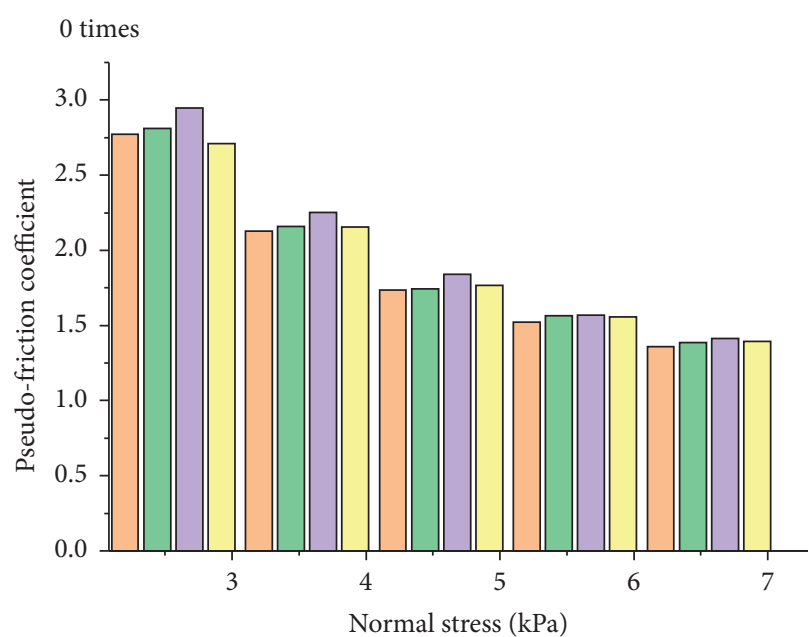

$\square$ 4\% water content
$\square$ 8\% water content

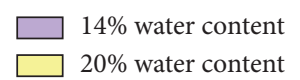

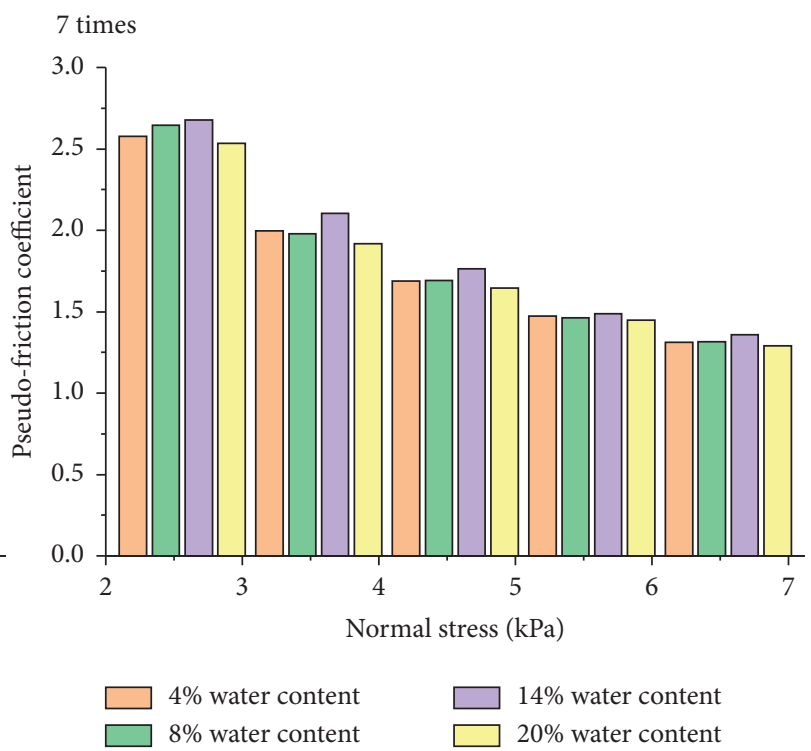

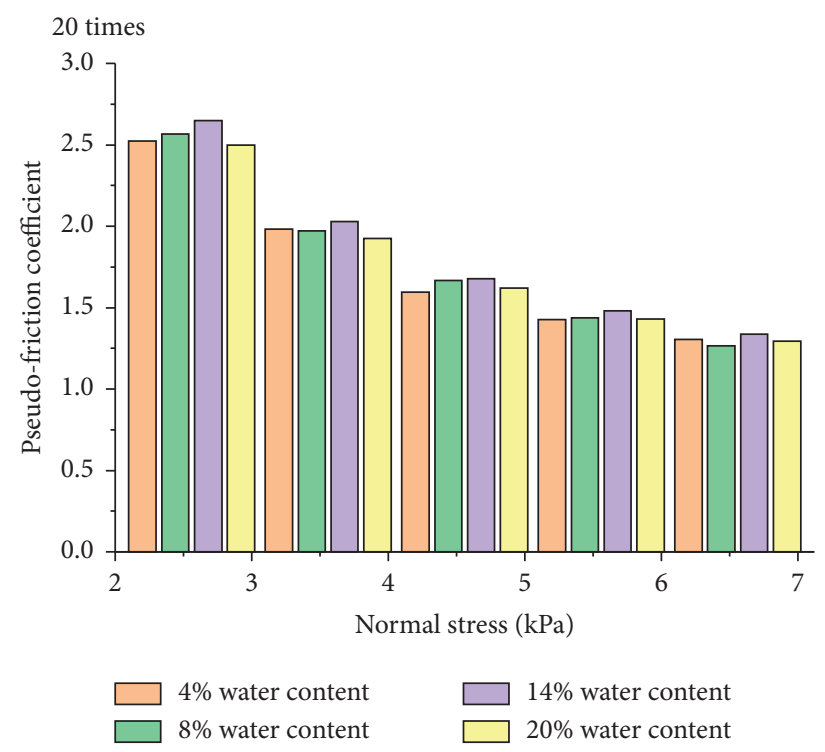

FiguRe 17: Variation of the apparent friction coefficient of the reinforced soil interface with different cycles.

coefficient of the reinforced soil decreases with the increase of the times of the dry-wet cycle. The difference between the interface friction coefficients after 7 times and 30 times is not obvious. After 7 times of the dry-wet cycle, the continued increase in the times of the cycle has little effect on the interface friction coefficient of the reinforced soil. When the normal stress is the smallest, the interface friction coefficient of the reinforced soil after 0 times of the dry-wet cycle is 2.95; the interface friction coefficient after 7 times of the dry-wet cycle is 2.68 , which is reduced by $9.2 \%$; the interface friction coefficient after 30 times of the dry-wet cycle is 2.65 , which is reduced by $10.2 \%$; this shows that the effect of the reinforcement of the fill and the geogrid will decrease after the dry-wet cycle of the reinforced soil. Combined with the results of the scanning electron microscope image analysis, the fractal dimension of the morphological distribution of the reinforced soil particles gradually increases with the increase of the times of the dry-wet cycle. The larger the fractal dimension, the lower the roughness of the particles. Under the action of water migration, the abundance of the soil particles gradually increases and the particles are rounded. Multi-parameters indicate that the frictional resistance between the reinforcement and the soil and between the soil particles becomes weaker, which is manifested in the macroscopic view that the friction coefficient of the reinforcement and the soil interface decreases with the increase of the times of the dry-wet cycle.

4.4. Analysis of the Variation of Cohesion. The dry-wet cycle test and the pull-out test can obtain the cohesion of the reinforced soil under different working conditions. The variation of the cohesion of the reinforced soil with the times of the dry-wet cycle is shown in Figure 19.

The cohesion of the reinforced soil decreases with the increase of the times of the dry-wet cycle. The difference 


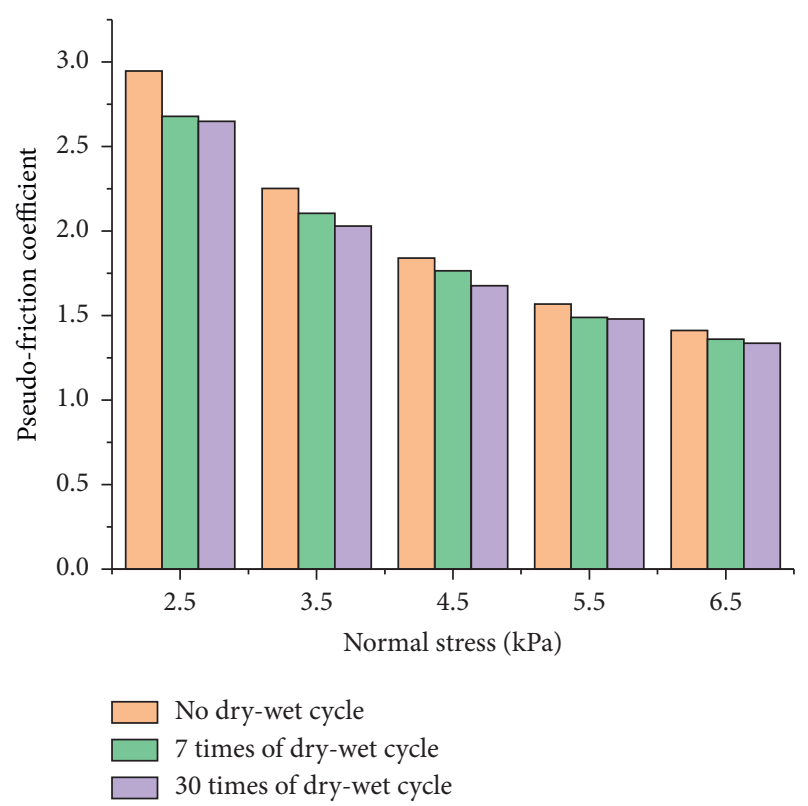

FIGURE 18: Variation of friction coefficient at the interface of $14 \%$ water content at the different cycle times.

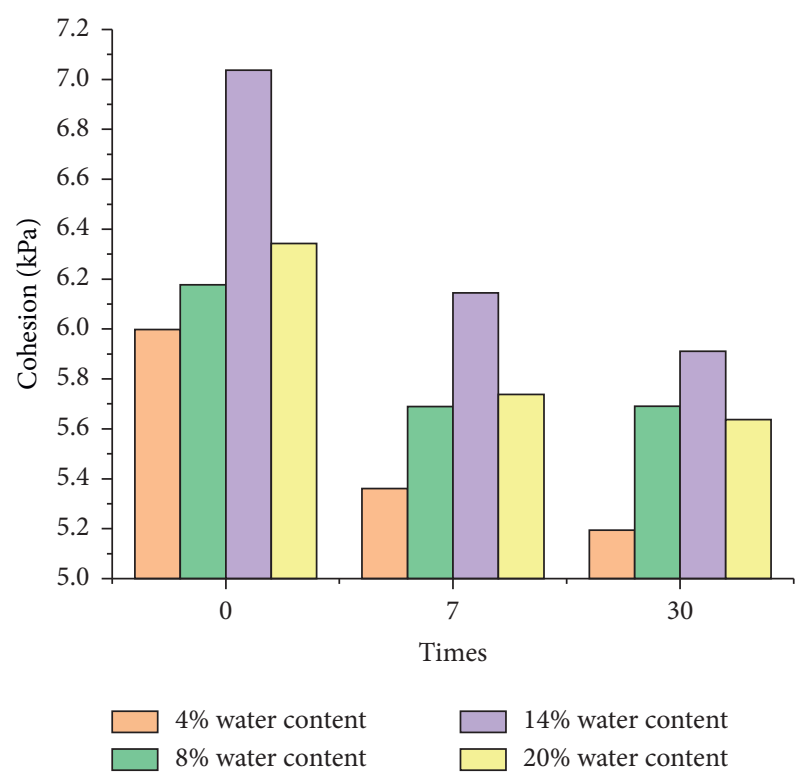

FIGURE 19: Variation of the cohesion of the reinforced soil with the times of the cycle under different water contents.

between the cohesion after 7 times of the dry-wet cycle and 30 times is about $0.2 \mathrm{kPa}$, which means that after 7 times of the dry-wet cycle, the times of dry-wet cycle continued to increase and has little effect on the cohesion of the reinforced soil; the cohesion of the reinforced soil without the dry-wet cycle is $7.0370 \mathrm{kPa}$, and the cohesion after 7 times of the drywet cycle is $6.1451 \mathrm{kPa}$, which reduced by $12.7 \%$.

Cohesion is the mutual attraction between adjacent soil bodies. The contact area of the reinforced soil particles becomes smaller under the action of the dry-wet cycle, and water migration also dissolves and entrains some particles, which reduces the adhesion between the soil particles; On the one hand, polar water molecules are adsorbed under the action of electrostatic gravity on the particle surface,; the combined water film is thickened, the distance between the particles increases, and the molecular gravity decreases, so that the interaction force between adjacent molecules gradually decreases, and the cohesion of the soil decreases. After 30 times of dry-wet cycle, the cohesion of the soil does not decrease significantly, because the soil has formed a stable seepage channel, thus the cohesion basically does not continue to decrease as times of cycle increase.

It can be seen from Figure 19 that the cohesion of the reinforced soils with different water contents shows a nonlinear decreasing trend with the increase of the times of the dry-wet cycle, and the water content has little effect on the change trend of the cohesion of the reinforced soils with the times of the dry-wet cycle. The cohesion of the reinforced soil is greatly reduced after 7 times of the dry-wet cycle, and the rate of decrease becomes slower after 30 times of the drywet cycle. After 30 times of the dry-wet cycle, the cohesion of the reinforced soil with a water content of $4 \%$ was the smallest at $5.19 \mathrm{kPa}$, and the cohesion of the reinforced soil with a water content of $14 \%$ was the largest at $5.91 \mathrm{kPa}$, which was significantly greater than that in the dry state. The values of the reinforced soil's cohesion from large to small correspond to water contents of $14 \%, 20 \%, 8 \%$, and $4 \%$, respectively. The cohesion of the reinforced soils with water contents of $8 \%$ and $20 \%$ are similar.

4.5. The Influence of the Dry-Wet Cycles on the Stability of the Retaining Structure. The strength reduction method [29] is used to analyze the stability of the reinforced soil slopes and the plain soil slopes under the action of the dry-wet cycles. In this paper, the FLAC3D [30] software is used for numerical simulation, and the factors of safety of the two types of slopes in different times of the dry-wet cycle are deduced. Figure 20 shows the variation of the factor of safety of the reinforced soil slopes and the plain soil slopes with the times of the drywet cycle.

It can be seen from Figure 20 that the factor of safety of the slope shows a nonlinear decreasing trend with the increase of the times of the dry-wet cycle. Without the dry-wet cycle, the factor of safety of the reinforced soil slope is 2.87 , and that of the plain soil slope is 2.23 . When the dry-wet cycle is 7 times, the factor of safety of the reinforced soil slope is 2.24 , and the factor of safety of the plain soil slope is 1.76 , and the factor of safety is reduced greatly. When the dry-wet cycle is 30 times, the factor of safety of the reinforced soil slope is 1.94 , the factor of safety of the plain soil slope is 1.35 , and the reduction of the factor of safety becomes smaller. Among them, the factor of safety of the reinforced soil slopes is always higher than that of the plain soil slopes, and after 30 wetdry cycles, the factor of safety of the reinforced soil slopes has dropped by $32.4 \%$, and the factor of safety of the plain soil slopes has dropped by $39.5 \%$. It shows that the drywet cycle will reduce the stability of the slope, and the stability of the reinforced soil slope under the dry-wet cycle is better than that of the plain soil slope. 


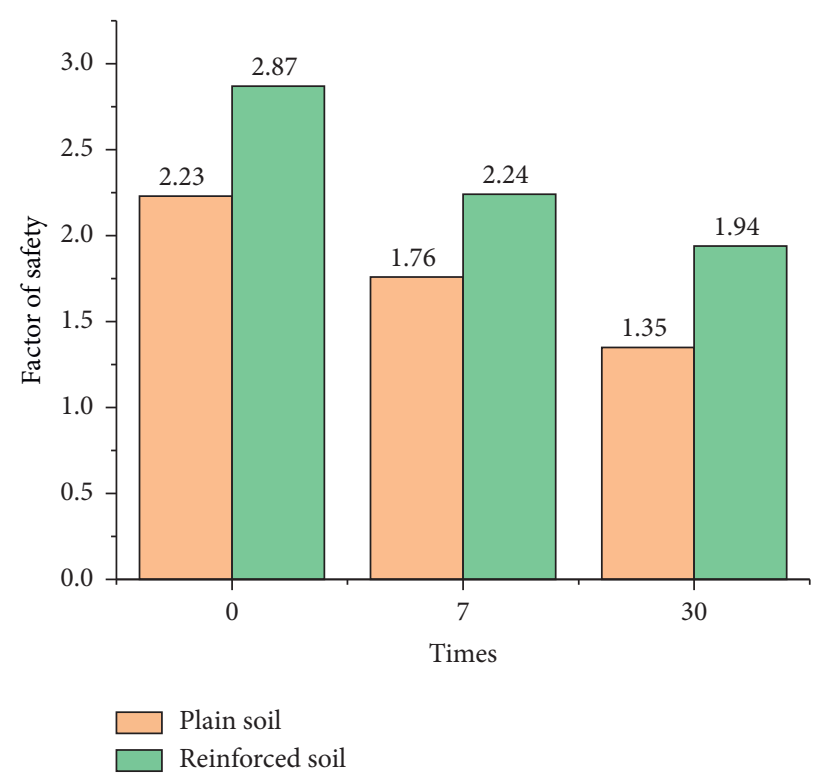

FIgURE 20: Variation law of the factor of safety of the reinforced soil slope and the plain soil slope.

\section{Conclusion}

Based on the results of the dry-wet cycle test and the pull-out test, the influence of the times of the dry-wet cycle on the microstructure changes of the reinforced soil and the mechanical properties of the interface between the reinforced materials and soils was studied. After analyzing relevant test data, the following conclusions are drawn:

(1) As the times of the dry-wet cycle increases, the contact area between the reinforced materials and the soil particles gradually decreases, and the contact form develops from surface-surface contact to mainly edge-edge or edge-surface contact. The pore area and the number of pores in the reinforced soil gradually increase with the increase in the times of the dry-wet cycle. The number of micropores and small pores increases more, so that the proportion of micropores and small pores increases, and the proportion of large and medium pores decreases.

(2) With the increase in the times of the dry-wet cycle, the ratio of the long axis to the short axis of the reinforced soil particles decreases under the action of water migration, the abundance value gradually rises, and the fractal dimension value of the shape distribution gradually rises too. It shows that the reinforced soil particles gradually become rounded and their surface roughness decreases under the action of the dry-wet cycle.

(3) When the times of the dry-wet cycle is the same, the ultimate pull-out force of the reinforced soil increases linearly with the increase of the overburden pressure; when the overburden pressure is the same, the ultimate pull-out force of the reinforced soil decreased with the times of the dry-wet cycle. When the overburden pressure is $5 \mathrm{kPa}$ and the water content is $14 \%$, the ultimate pull-out force of the reinforced soil is reduced by $5.3 \%$ after 30 times of the dry-wet cycle. The dry-wet cycle causes the porosity of the soil to increase, the abundance of the soil particles increases, and the occlusal force of the interface between the reinforced materials and the soils is weakened, which causes a decrease in the ultimate pull-out force of the reinforced soil in a macroscopic view.

(4) The shear stress of the reinforced soil is linearly related to the normal stress. The shear stress and the normal stress of the reinforced soil are linearly fitted. The fitting index is between 0.9693 and 0.9959 . The fitting effect is good and conforms to the Coulomb law.

(5) When the normal stress and the water content are constant, the friction coefficient of the reinforced soil interface decreases with the increase of the times of the dry-wet cycle. When the normal stress is $1 \mathrm{kPa}$ and the water content is $14 \%$, the interfacial friction coefficient of the reinforced soil after 30 times of the dry-wet cycle is reduced by $10.2 \%$. After the reinforced soil undergoes the dry-wet cycle, the effect of the soils and the geogrids will decrease. The dry-wet cycle leads to the abundance of the soil particles to increase, the fractal dimension of particle morphology distribution increases, the surface roughness of the particles decreases, and the friction resistance between particles decreases, which causes a macroscopic decrease in the friction coefficient of the reinforced soil interface.

(6) When the normal stress and water content are constant, the cohesion of the reinforced soil shows a nonlinear decrease trend with the increase of the times of the dry-wet cycle. After a certain times of the dry-wet cycle of the reinforced soil; the contact area between the soil particles decreases; the number of 
pores, especially the number of micropores and small pores, increases; the combined water film between the particles increases; and the interaction force between adjacent molecules decreases, which causes a macroscopic decrease in the cohesion of the reinforced soil.

(7) Under the action of the dry-wet cycles, the calculated factor of safety of the reinforced soil slopes is higher than that of the plain soil slopes, indicating that reinforcement in the soil can improve the stability of the slope. However, as the times of the dry-wet cycle increases, the stability of the reinforced soil slopes will also decrease to a certain extent.

\section{Data Availability}

The datasets generated during the current study are available from the corresponding author on reasonable request.

\section{Conflicts of Interest}

The authors declare that they have no conflicts of interest.

\section{Acknowledgments}

This study was supported by the National Key Research and Development Program of China (Grant no. 2016YFE0205100).

\section{References}

[1] J. Zhang, Q. Xu, and D. Sun, "Simulation of soil-water characteristic curves during drying and wetting cycles," Rock and Soil Mechanics, vol. 35, no. 3, pp. 689-695, 2014.

[2] H. Nowamooz and F. Masrouri, "Relationships between soil fabric and suction cycles in compacted swelling soils," Engineering Geology, vol. 114, no. 3-4, pp. 444-455, 2010.

[3] A. Aldaood, M. Bouasker, and M. Al-Mukhtar, "Impact of wetting-drying cycles on the microstructure and mechanical properties of lime-stabilized gypseous soils," Engineering Geology, vol. 174, pp. 11-21, 2014.

[4] R. Zhang, H. Yang, and J. Zheng, "The effect of vertical pressure on the deformation and strength of expansive soil during cyclic wetting and drying," in Proceedings of the Fourth International Conference on Unsaturated Soils, Carefree, Arizona, April 2006.

[5] L.-w. Kong, M. Wang, A.-g. Guo et al., "Effect of drying environment on engineering properties of an expansive soil and its microstructure," Journal of Mountain Science, vol. 14, no. 6, pp. 1194-1201, 2017.

[6] M. H. T. Wang, E. K. Yanful, and A. Fakher, "Physical modeling of desiccation cracking in plastic soils," Engineering Geology, vol. 97, no. 1-2, pp. 25-31, 2008.

[7] M. Julina and T. Thyagaraj, "Quantification of desiccation cracks using X-ray tomography for tracing shrinkage path of compacted expansive soil," Acta Geotechnica, vol. 14, no. 1, pp. 35-56, 2019.

[8] J. Xiong, B. Liu, L. Liu et al., "A method for crack image processing of red clay," Chinese Journal of Underground Space and Engineering, vol. 16, no. 2, pp. 381-387, 2020.

[9] Yang H, Y. Huang, D. Zhou et al., "The effect of dryingwetting cycle on the permeability of yunnan laterite," Science Technology and Engineering, vol. 19, no. 27, pp. 289-297, 2019.
[10] B. A. Albrecht and C. H. Benson, "Effect of desiccation on compacted natural clays," Journal of Geotechnical and Geoenvironmental Engineering, vol. 127, no. 1, pp. 67-75, 2001.

[11] M. Ziegler, "Application of geogrid reinforced constructions: history, recent and future developments," Procedia Engineering, vol. 172, pp. 42-51, 2017.

[12] G. T. Mehrjardi, R. Behrad, and S. N. M. Tafreshi, "Scale effect on the behavior of geocell-reinforced soil," Geotextiles and Geomembranes, vol. 47, no. 2, pp. 154-163, 2019.

[13] Y. Kurakami, Y. Nihei, M. Morita et al., "Effect of river levee with geosynthetic-reinforced soil against overflow erosion and infiltration," in Proceedings of the 6th International Symposium on Hydraulic Structures: Hydraulic Structures and Water System Management, pp. 285-294, Portland, OR, USA, 2016.

[14] J. Xiao, H. P. Yang, and H. Fu, "Comparison model test on effect of geogrid reinforcement for expansive soil slope," China Journal of Highway and Transport, vol. 27, no. 7, pp. 24-31, 2014.

[15] D. J. U. Infante, G. M. A. Martinez, P. A. Arrua et al., "Shear strength behavior of different geosynthetic reinforced soil structure from direct shear test," International Journal of Geosynthetics and Ground Engineering, vol. 2, no. 2, pp. 1-16, 2016.

[16] K.-H. Yang, J. N. Thuo, J.-W. Chen, and C.-N. Liu, "Failure investigation of a geosynthetic-reinforced soil slope subjected to rainfall," Geosynthetics International, vol. 26, no. 1, pp. 42-65, 2019.

[17] R. Liu, M. Saberian, J. Li, G. Shams, and P. v. Gelder, "Properties of geogrid-reinforced marine slope due to the groundwater level changes," Marine Georesources \& Geotechnology, vol. 36, no. 6, pp. 735-748, 2018.

[18] F. Shams, G. Li, Y. Mu et al., "Experimental study of deformation characteristics of compacted loess subjected to drying-wetting cycle," Rock and Soil Mechanics, vol. 37, no. 8, pp. 2306-2312, 2016.

[19] H. Deng, Y. Xiao, J. Fang et al., "Shear strength degradation and slope stability of soils at hydro-fluctuation belt of river bank slope during drying-wetting cycle," Rock and Soil Mechanics, vol. 38, no. 9, pp. 2629-2638, 2017.

[20] S. Yang and W. Liu, "Application of image-pro plus in the shear strength and micro-structure of solidified soil mixed with fly ash," Multimedia Tools and Applications, vol. 79, pp. 10065-10075, 2019.

[21] W. Ye, Y. Wu, G. Yang et al., "Study on micro-structure and macro-mechanical properties of paleosol under dry-wet cycles," Chinese Journal of Rock Mechanics and Engineering, vol. 38, no. 10, pp. 2126-2137, 2019.

[22] Z. Yuan, Research on Change Mechanism of Strength and Micro-structure of Loess under Wetting-Drying Cycle, Chang'an University, Xi'an, China, 2015.

[23] X. Lei, "Pore types and collapsibility of loess in China," Science in China Series B, vol. 11, no. 12, pp. 1398-1411, 1987.

[24] X. Zhang and C. Wang, "Microstructural change of soft clay before and after one-dimensional compression creep," Chinese Journal of Geotechnical Engineering, vol. 32, no. 11, pp. 1688-1694, 2010.

[25] G. Huang and W. Zhan, "Fractal property of soil particle size distribution and its application," Acta Pedologica Sinica, vol. 4, no. 4, pp. 490-497, 2002.

[26] C. A. Moore and C. F. Donaldson, "Quantifying soil microstructure using fractals," Géotechnique, vol. 45, no. 1, pp. 105-116, 1995. 
[27] D. Zhang and H. Rong, "Analysis on large scale pull-out model test of reinforced coarse-grained soil," Hebei Journal of Industrial Science and Technology, vol. 37, no. 3, pp. 196-202, 2020.

[28] R. Chen, B. Li, D. Hao et al., "Simulation for interaction between geogrid and soil by cohesive zone model," Chinese Journal of Geotechnical Engineering, vol. 42, no. 5, pp. 934940, 2020.

[29] H. Rafiei Renani and C. D. Martin, "Factor of safety of strainsoftening slopes," Journal of Rock Mechanics and Geotechnical Engineering, vol. 12, no. 3, pp. 473-483, 2020.

[30] L. Zabuski, "Three-Dimensional analysis of a landslide process on a slope in carpathian flysch," vol. 66, no. 1-2, pp. $27-45,2019$. 